\title{
Breast Cancer Primary Prevention and Diet: An Umbrella Review
}

\author{
Alessandra Buja ${ }^{1, *(D)}$, Marco Pierbon ${ }^{2}$, Laura Lago ${ }^{2}$, Giulia Grotto ${ }^{1}$ and Vincenzo Baldo $^{1}$ (D) \\ 1 Department of Cardiological, Thoracic and Vascular Sciences, University of Padova, Via Loredan 18, \\ 35127 Padova, Italy; giulia.grotto.1@studenti.unipd.it (G.G.); vincenzo.baldo@unipd.it (V.B.) \\ 2 Masters Course in Sciences for the Public Health and Prevention Professions, University of Padova, \\ Via Giustiniani 2, 35127 Padova, Italy; marco.pierbon94@gmail.com (M.P.); laura.lago@unipd.it (L.L.) \\ * Correspondence: alessandra.buja@unipd.it; Tel.: +39-82-75-387; Fax: +39-82-75-392
}

Received: 2 June 2020; Accepted: 25 June 2020; Published: 1 July 2020

check for updates

\begin{abstract}
Introduction: Many studies have been published, but none have pooled the useful evidence available in the literature to produce guidelines and health policies promoting healthy eating styles to prevent breast cancer (BC). The present study aimed to summarize the evidence produced to date, taking a judicious, critical approach to the quality of the studies analyzed. Methods: An umbrella review method was adopted, which is a systematic review of second-level studies, meta-analyses and literature reviews. Results: In all, 48 studies were considered: 32 meta-analyses, 4 pooled analyses, 5 systematic reviews, and 7 qualitative reviews. A higher intake of total meat, or red or processed meats, or foods with a high glycemic index, or eggs would seem to be associated with a higher risk of BC. Some foods, such as vegetables, would seem instead to have an inverse association with BC risk. One meta-analysis revealed an inverse association between citrus fruit and mushroom consumption and BC. Some nutrients, such as calcium, folate, vitamin D, lignans and carotenoids, also seem to be inversely associated with $\mathrm{BC}$ risk. The evidence is still conflicting as concerns exposure to other dietary elements (e.g., polyunsaturated fatty acids, dairy foods). Conclusion: Nutrition is one of the most modifiable aspects of people's lifestyles and dietary choices can affect health and the risk of cancer. Overall, adhering to a healthy eating style may be associated with a significant reduction in the risk of $\mathrm{BC}$.
\end{abstract}

Keywords: diet; cancer risk; breast cancer; cancer prevention; dietary intervention

\section{Introduction}

Breast cancer (BC) is the most frequently diagnosed female cancer, accounting for $29 \%$ of cancers in women [1]. It is important for primary prevention to include reducing modifiable risk factors, such as obesity, a sedentary lifestyle, and a poor diet. Each of these factors can have various effects, depending on breast tissue type and age (premenopausal and menopausal).

As concerns diet, the role of alcohol as a risk factor has been well established: the results of the 2011 EPIC (European Prospective Investigation into Cancer and Nutrition) survey found that 5\% of BCs could be attributable to alcohol consumption [2]. Numerous studies have addressed the role of several other foods too, sometimes reporting divergent results. For example, according to the EPIC survey, a diet rich in saturated fat is associated with a higher risk of estrogen- and progesterone-positive cancer, with a significantly higher hazard ratio [3]. Similar results emerged from a Swedish cohort survey, in which a high dietary fat consumption seemed to lead to a significant increase in the risk of developing BC [4]. On the other hand, a case-control survey conducted in China in 2008 found no significant association between the various types of dietary fat and the odds of cancer [5]. Red meat and animal proteins seem to be associated with an increase in the risk of neoplastic disease: Jansen 
suggested that consuming them in large amounts anticipates menarche, and this is recognized as a risk factor and predictor of $\mathrm{BC}[6]$.

Several epidemiological studies have shown that consuming soy products is associated with a lower incidence of hormone-related tumors, including BC, due to the properties of isoflavones and phytoestrogens [7-9].

Despite the many studies conducted on the topic, the evidence in the literature has yet to be pooled and used for the purpose of drawing up guidelines and health policies to promote healthy eating styles. The aim of the present work was thus to obtain a synthesis of the scientific evidence produced over the last 15 years regarding the association between nutrition and BC. The umbrella review approach was adopted, which entails conducting a systematic review of second-level studies, meta-analyses, and literature reviews, and summarizing the evidence available to date in the light of a judicious critical assessment of the quality of the studies involved.

\section{Materials and Methods}

\subsection{Study Design}

An umbrella review was conducted, which involved critically examining the literature, starting from a synthesis of the previously-published second-level research, and performing a critical review of all available evidence [10,11].

\subsection{Sources}

A comprehensive search (see Figure 1) was conducted in the PubMed (Medline) and Scopus databases using combined MeSH terms: ("breast neoplasm" (MeshTerm) OR "breast cancer" OR "breast tumor") AND ("diet" (MeshTerm) OR "alimentation" OR "nutrition") AND ("prevention" (MeshTerm) OR "risk" (MeshTerm) OR "association" (MeshTerm)) from 2000 to February 2016. The search concerned meta-analyses, pooled analyses, systematic reviews, and qualitative reviews on the risk of $\mathrm{BC}$ in the female population. We checked the references for the systematic reviews and meta-analyses retrieved, and the proceedings of relevant conferences for articles missed by the electronic search. In particular, we included studies that examined the presence and intake of foods in the diet (exogenous exposure), instead of just measuring endogenous nutrients. Studies with the subsequent characteristics were excluded:

1. General and descriptive reports, comments and updates without any reported association measures;

2. Studies on populations or groups at increased risk;

3. Analyses of dietary supplements or studies examining the combined effect of physical activity and diet;

4. Studies involving $\mathrm{BC}$ recurrence;

5. Studies written in languages other than Italian or English.

\subsection{Data Extraction}

We first extracted the general characteristics of each eligible systematic review or meta-analysis, the first author's name, the year of publication, the type of epidemiological design, the total size of the samples in all the studies included in a review. Then, we extracted the main findings of each study, including how exposure was measured and the overall results, and we summarized the meta-analytic estimates. Finally, we input details of the limitations of the study, and the authors' recommendations. Two investigators (M.P., L.L.) independently searched the literature, assessed the eligibility of the papers they retrieved, and extracted the data. Disagreements were solved by discussion with a third investigator (A.B.). 


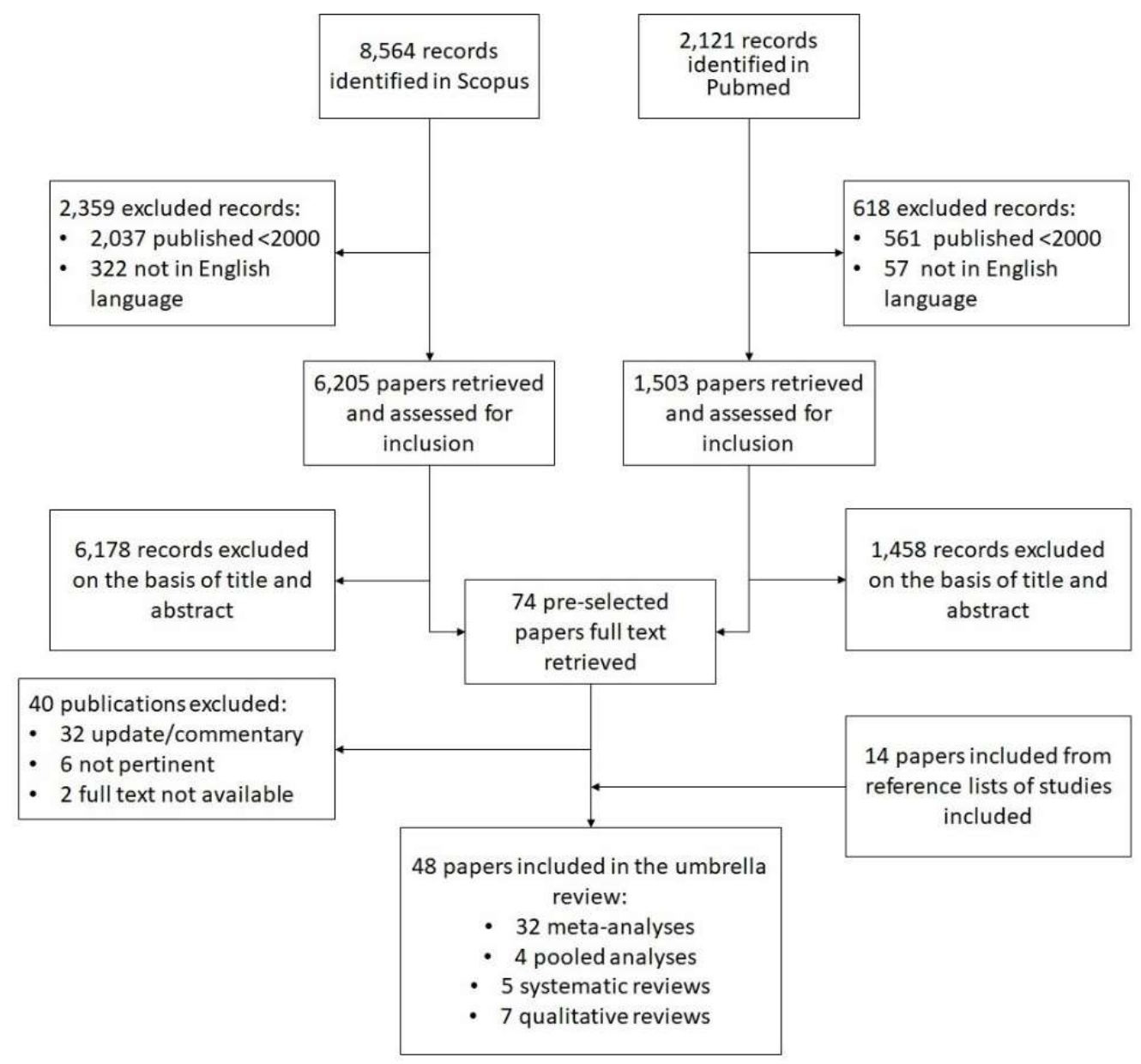

Figure 1. The research strategy used to identify the articles for the umbrella review.

\subsection{Study Quality Assessment}

The PRISMA (preferred reporting items for systematic reviews and meta-analyses) checklist, developed in 2009 [12,13], was used for a critical assessment of the internal validity of the reviews and meta-analyses considered. A checklist was compiled for each study included in the umbrella review (see Table S3).

\section{Results}

Our literature search identified 8564 results in Scopus and 2121 in PubMed (Figure 1). Then 2359 of the former studies and 618 of the latter were removed after applying publication and language filters. Another 6178 and 1503 studies were excluded after screening the abstracts and titles. In the end, 74 studies were included in the pre-selection phase, and 40 of them were deleted after reading the full text, leaving 34 studies. At this point, 14 further articles were drawn from the lists of references of the reviews considered. A total of 48 studies were tabulated for the purposes of our umbrella review: 32 meta-analyses, 4 pooled analyses, 5 systematic reviews, and 7 other reviews. The quality of these studies varied considerably: the meta-analyses scored a mean 20.1 points on the PRISMA checklist, the pooled analyses 15.5, the systematic reviews 15.2, and the other reviews 9 points.

Table 1 contains data concerning the general characteristics of the studies identified, Table 2 shows the exposure measures and main findings of the studies, Table 3 shows the conclusions and recommendations, and Tables S1 and S2 show summaries of the results of the studies. 


\subsection{Dietary Patterns}

The umbrella review ultimately included five studies (two meta-analyses [14,15], one three-cohort analysis [16], two systematic reviews $[17,18])$ on the association between dietary patterns and BC risk. A diet was judged to be "healthy" if it included plenty of fiber and limited amounts of saturated fat, animal proteins, and refined sugars. The Mediterranean diet (MD) also contemplates a moderate consumption of red wine, and the use of extra-virgin olive oil for seasoning. These two (healthy and MD) eating patterns have been associated with a protective role against the development of $\mathrm{BC}[14,17,18]$ (Brennan, all studies combined: Odds Ratio (OR) 0.89, 95\% CI 0.82-0.99). A systematic review [18], which included five cohort studies and three case-control studies, found that the case-control studies had identified an inverse association between MD and BC risk, in both pre- and post-menopausal women, but the cohort studies reported controversial results. One study that examined three cohorts using a standardized method found no association between a vegetable-based diet (characterized by high intakes of vegetables, legumes, fruit, pasta, fish, and oil) and BC risk in any of the cohorts [16]. This goes to show that the literature still does not offer a clear evidence of the association between a healthy diet and the risk of $\mathrm{BC}$.

The Western diet includes large amounts of refined sugars, proteins, saturated fats, and alcohol, and-judging from case-control studies alone-it appears to be associated with a higher risk of BC (OR 1.31, 95\% CI 1.05-1.63 [14]). On the other hand, when Brennan combined all types of study, there was no evidence of any difference in the risk of $B C$ for women in the highest category of intake of Western/unhealthy dietary patterns compared with the lowest category (OR $=1.09,95 \%$ CI: 0.98, 1.22).

\subsection{Foods}

Five studies (two meta-analyses [19,20], one systematic review [21], one pooled analysis [22] and one qualitative review [23]) examined the association between $\mathrm{BC}$ risk and red and processed meat consumption. Guo's meta-analysis [19] found a positive association between the risk of BC and both red meat (summary RR 1.10, 95\% CI 1.02-1.19) and processed meat (summary RR $1.08,95 \%$ CI 1.01-1.15), and Taylor [20] reported an association between red meat consumption and a higher BC risk in premenopausal women (summary RR 1.24, 95\% CI 1.08-1.42) [24]. On the other hand, the pooled study [22] found no significant associations between the intake of total meat, red meat or white meat, and $\mathrm{BC}$ risk.

It emerged from one meta-analysis [25] that a high weekly rate of egg consumption was associated with an increased risk of developing BC, that was only significant in women of postmenopausal age (RR estimates: 1.06 ; 95\% CI 1.02-1.10).

Of the four studies that considered dairy food consumption (two meta-analyses [26,27], one pooled analysis [22], and one systematic review [28]), two reported an inverse association between the consumption of dairy products and the risk of BC [26,27].

Eleven of the studies analyzed the association between BC and the intake of soy foods, phytoestrogens, isoflavones, and lignans (six meta-analyses [9,29-33], three systematic reviews [22,28,34], and three qualitative reviews [35-37]). Three of these studies [29,32,34] demonstrated a significant inverse association between BC and a high lignans consumption in postmenopausal women, while two others [31,36] detected stronger evidence of this association in premenopausal women. The protective role of soy and phytoestrogens was recognized as significant in another seven studies $[9,21,28,30,33,35,37]$.

Seven studies (three meta-analyses [24,33,38], two systematic reviews [21,28], one qualitative review [23], and one pooled analysis [39]) investigated dietary fiber: the three meta-analyses confirmed its protective effect against BC, the pooled analysis [39] found an inverse borderline association, while one of the systematic reviews [21] and the qualitative review [23] found no association between fruit fiber intake and the incidence of $\mathrm{BC}$. The other systematic review only found a protective role for vegetable consumption, but no significant association with fruits or fruit fiber [28]. In another meta-analysis [40], Song reported a statistically significant association between citrus fruit consumption 
and the risk of developing BC (summary RR 0.90; 95\% CI 0.85-0.96). A further meta-analysis [41] showed a significant inverse association between mushroom consumption and the risk of BC, in both premenopausal women (RR 0.96, 95\% CI 0.91-1.00), and postmenopausal women (summary RR 0.94; 95\% CI 0.91-0.97). The scientific evidence of any role for green tea in preventing BC is rather weak: one meta-analysis [42] and one systematic review [28] included in our umbrella review found no significant association, while a subgroup analysis showed a statistically significant inverse association in case-control studies (summary RR 0.56; 95\% CI 0.38-0.83).

\subsection{Nutrients}

Eight of the studies (including four meta-analyses [33,43-45], two systematic reviews [21,28], one pooled analysis [46] and one qualitative review [23]) analyzed fat intake and the risk of BC. One meta-analysis [43] found no statistically significant association (summary RR estimate 1.11, 95\% CI 0.91-1.36); one of the systematic reviews [29] and the qualitative review [23] obtained similar results. Another four studies found an increased risk of BC in women with a high intake of total fat $[21,33,44]$ (Boyd et al. [44]) (summary RR 1.13, 95\% CI 1.03-1.25) and saturated fat [46] (summary RR 1.19, 95\% CI 1.00-1.19); one study found a positive association between a high intake of total fat and BC risk in postmenopausal women only [45] (summary RR 1.04, 95\% CI 1.01-1.07).

Only one of the two meta-analyses included in our review $[47,48]$ showed a modest increase in the risk of $\mathrm{BC}$ for women with dietary patterns associated with a high glycemic index (GI) or glycemic load (GL) [48]. Another two studies investigating the association between foods with a high GI or GL and the risk of $\mathrm{BC}$ also produced controversial results [21,28].

The consumption of polyunsaturated fatty acids (PUFA) was examined in seven studies (five meta-analyses [44,45,49-51], one systematic review [21], and one pooled analysis [46]). Two meta-analyses $[49,50]$ found a significant protective association, three studies $[44,46,51]$ reported a statistically insignificant inverse association, and another two studies [21,45] identified a higher risk of BC in women with a high PUFA consumption (summary RR: 1.07; 95\% CI 1.01-1.14).

As for vitamin D and calcium, three studies (two meta-analyses [52,53] and one qualitative review [54]) considered these factors, and their protective effect was confirmed.

Three studies (two meta-analyses [55,56], and one qualitative review [57]) tested the association between folate consumption and the risk of BC, finding no significant inverse association overall. The only study that considered flavonoids and flavones (one meta-analysis [58]) concluded that the risk of BC was significantly reduced in women with a high overall consumption of flavonoids (RR 0.88; 95\% CI 0.80-0.96), and flavones (RR 0.80; 95\% CI 0.76-0.91). One meta-analysis [59] and one systematic review [28] investigated the relationship between carotenoid consumption and BC: the meta-analysis found that $\alpha$-carotene and $\beta$-carotene consumption had a significant protective effect against $\mathrm{BC}$, while the systematic review showed no such association. One systematic review [28] studied the association between BC and vitamins A, B, C and E, finding no such association in ten cohort studies; a positive association between vitamin $\mathrm{E}$ and $\mathrm{BC}$ emerged in one cohort study.

The overall results of the studies are summarized in Tables S1 and S2. 
Table 1. Characteristics of studies included in the umbrella review.

\begin{tabular}{|c|c|c|c|c|c|c|c|}
\hline Author_Year & $\begin{array}{l}\text { Continent or Region, } n \text {. } \\
\text { of Studies }\end{array}$ & $\begin{array}{l}\text { Search Range } \\
\text { Applied }\end{array}$ & $\begin{array}{c}\text { Total n. of } \\
\text { Studies Included } \\
\text { in the Review }\end{array}$ & $\begin{array}{c}\text { Total Sample Size of all } \\
\text { Studies Included in the } \\
\text { Review }\end{array}$ & Type of Study & Study Design & $\begin{array}{l}\text { Association/s } \\
\text { Examined }\end{array}$ \\
\hline \multicolumn{8}{|c|}{ Characteristics of meta-analyses included in the umbrella review (32) } \\
\hline $\begin{array}{c}\text { Alexander } 2010 \\
{[43]}\end{array}$ & // & up to 2008 & $\begin{array}{l}11 \text { studies } \\
\text { referring to } 6 \\
\text { cohort studies }\end{array}$ & // & $\begin{array}{l}\text { meta-analysis and } \\
\text { review of } \\
\text { epidemiological } \\
\text { cohort studies }\end{array}$ & 6 cohort studies & $\begin{array}{c}\text { animal fat intake } \\
\text { and breast cancer } \\
\text { (BC) }\end{array}$ \\
\hline Aune 2012 [38] & $\begin{array}{l}9 \text { studies in America, } 6 \text { in } \\
\text { Europe and } 1 \text { in Asia }\end{array}$ & $\begin{array}{l}\text { from January } 2006 \\
\text { to } 31 \text { August } 2011\end{array}$ & $\begin{array}{l}16 \text { prospective } \\
\text { studies (17 } \\
\text { publications) }\end{array}$ & $\begin{array}{l}26,523 \text { cases among } \\
999,271 \text { participants }\end{array}$ & $\begin{array}{l}\text { systematic review } \\
\text { and meta-analysis }\end{array}$ & 16 cohort studies & $\begin{array}{c}\text { high vs. low } \\
\text { dietary fiber intake } \\
\text { and BC risk }\end{array}$ \\
\hline Boyd 2003 [44] & $\begin{array}{l}22 \text { studies in Europe } \\
\text { (including Russia), } 15 \text { in } \\
\text { North America, } 5 \text { in Asia, } \\
2 \text { in Australia and } 1 \text { in } \\
\text { South America (Uruguay) }\end{array}$ & $\begin{array}{c}\text { from January } 1966 \\
\text { to July } 2003\end{array}$ & 45 studies & $\begin{array}{c}16,280 \mathrm{BC} \text { cases and } 18,966 \\
\text { controls in case-control } \\
\text { studies; } 8735 \mathrm{BC} \text { cases out } \\
\text { of } 568,549 \text { participants } \\
\text { (total } 25,015 \mathrm{BC} \text { cases and } \\
580,000 \text { controls) }\end{array}$ & $\begin{array}{l}\text { meta-analysis of } \\
\text { the published } \\
\text { literature }\end{array}$ & $\begin{array}{c}31 \text { case-control } \\
\text { studies and } 14 \\
\text { prospective studies } \\
\text { (cohort and nested } \\
\text { case-control studies) }\end{array}$ & $\begin{array}{l}\text { dietary fat and } \mathrm{BC} \\
\text { risk }\end{array}$ \\
\hline Brennan $2010[14]$ & $\begin{array}{c}7 \text { studies in North } \\
\text { America, } 5 \text { in Europe, } 2 \text { in } \\
\text { South America (Uruguay), } \\
2 \text { in Asia }\end{array}$ & $\begin{array}{l}\text { up to November } \\
2009\end{array}$ & 16 articles & $\begin{array}{l}\text { 13,885 BC cases out of } \\
465,891 \text { participants in } \\
\text { cohort studies; } 10,746 \mathrm{BC} \\
\text { cases and } 34,311 \text { controls } \\
\text { in case-control studies }\end{array}$ & $\begin{array}{l}\text { systematic review } \\
\text { and meta-analysis }\end{array}$ & $\begin{array}{c}8 \text { case-control and } 8 \\
\text { cohort studies }\end{array}$ & $\begin{array}{l}\text { dietary patterns } \\
\text { and } B C \text { risk }\end{array}$ \\
\hline Buck 2010 [29] & $\begin{array}{l}16 \text { studies in Europe, } 6 \text { in } \\
\text { North America } 1 \text { in Asia } \\
\text { and } 1 \text { in Australia }\end{array}$ & $\begin{array}{l}\text { from } 1997 \text { to } \\
\text { August } 2009\end{array}$ & $\begin{array}{c}24 \text { articles, } \\
\text { including } 21 \\
\text { studies (all on } \\
\text { lignan } \\
\text { exposures-9 } \\
\text { studies on } \\
\text { calculated dietary } \\
\text { plant lignans) }\end{array}$ & $\begin{array}{c}\text { 21,159 BC cases among } \\
\text { 188,302 participants } \\
\text { overall }\end{array}$ & meta-analysis & $\begin{array}{c}11 \text { cohort studies } \\
\text { and } 10 \text { case-control } \\
\text { studies }\end{array}$ & $\begin{array}{l}\text { lignans and } B C \\
\text { risk }\end{array}$ \\
\hline Chen 2010 [52] & // & up to July 2009 & $\begin{array}{c}11 \text { studies on } \\
\text { vitamin } D \text { intake } \\
\text { and } 15 \text { on calcium } \\
\text { intake ( } 26 \text { studies } \\
\text { in all) }\end{array}$ & // & meta-analysis & $\begin{array}{c}5 \text { case-control and } 6 \\
\text { cohort studies on } \\
\text { vitamin D intake; } 9 \\
\text { case-control and } 6 \\
\text { cohort studies on } \\
\text { calcium intake }\end{array}$ & $\begin{array}{c}\text { vitamin } \mathrm{D} \text { and } \\
\text { calcium intake on } \\
\mathrm{BC} \text { risk }\end{array}$ \\
\hline
\end{tabular}


Table 1. Cont.

\begin{tabular}{|c|c|c|c|c|c|c|c|}
\hline Author_Year & $\begin{array}{c}\text { Continent or Region, } \mathbf{n} \text {. } \\
\text { of Studies }\end{array}$ & $\begin{array}{l}\text { Search Range } \\
\text { Applied }\end{array}$ & $\begin{array}{c}\text { Total n. of } \\
\text { Studies Included } \\
\text { in the Review }\end{array}$ & $\begin{array}{c}\text { Total Sample Size of all } \\
\text { Studies Included in the } \\
\text { Review }\end{array}$ & Type of Study & Study Design & $\begin{array}{l}\text { Association } / \mathrm{s} \\
\text { Examined }\end{array}$ \\
\hline Chen 2014 [55] & $\begin{array}{l}20 \text { studies in North } \\
\text { America,10 in Europe, } 9 \text { in } \\
\text { Asia, } 1 \text { in Australia, } 1 \text { in } \\
\text { China } 1 \text { in Brazil }\end{array}$ & up to January 2014 & $\begin{array}{l}50 \text { eligible studies, } \\
42 \text { of which } \\
\text { reported on folate } \\
\text { intake and breast } \\
\text { cancer }\end{array}$ & $\begin{array}{c}26,205 \mathrm{BC} \text { cases out of } \\
744,068 \text { participants in } \\
\text { prospective studies; } \\
16,826 \mathrm{BC} \text { cases and } 21,820 \\
\text { controls in case-control } \\
\text { studies }\end{array}$ & $\begin{array}{l}\text { systematic review } \\
\text { and meta-analysis }\end{array}$ & $\begin{array}{l}16 \text { prospective } \\
\text { studies (14 cohort } \\
\text { and } 2 \text { nested } \\
\text { case-control) on } \\
\text { folate intake; } 26 \\
\text { case-control studies } \\
\text { on folate intake (8 } \\
\text { studies on blood } \\
\text { folate levels) }\end{array}$ & $\begin{array}{c}\text { folate intake and } \\
\text { overall BC risk }\end{array}$ \\
\hline Dong 2011 (a) [29] & $\begin{array}{c}8 \text { studies in Europe, } 4 \\
\text { studies in Asia, } 2 \text { studies } \\
\text { in USA }\end{array}$ & $\begin{array}{l}\text { up to September } \\
2010\end{array}$ & $\begin{array}{l}14 \text { studies on } B C \\
\text { incidence }\end{array}$ & $\begin{array}{c}369,934 \text { participants and } \\
5828 \text { cases of BC }\end{array}$ & $\begin{array}{l}\text { meta-analysis of } \\
\text { prospective } \\
\text { studies (nested } \\
\text { case-control and } \\
\text { cohort studies) } \\
\end{array}$ & $\begin{array}{c}6 \text { nested case control } \\
\text { studies and } 8 \text { cohort } \\
\text { studies }\end{array}$ & $\begin{array}{c}\text { soy isoflavone } \\
\text { consumption anc } \\
\text { BC risk }\end{array}$ \\
\hline Dong 2011 (b) [25] & $\begin{array}{l}9 \text { studies in USA, } 8 \text { in } \\
\text { Europe, } 1 \text { in Japan }\end{array}$ & up to January 2011 & 18 studies & $\begin{array}{l}24,187 \mathrm{BC} \text { cases and } \\
1,063,471 \text { participants }\end{array}$ & $\begin{array}{l}\text { meta-analysis of } \\
\text { prospective } \\
\text { studies (nested } \\
\text { case-control and } \\
\text { cohort studies) } \\
\end{array}$ & $\begin{array}{l}18 \text { cohort studies; } 12 \\
\text { studies on total dairy } \\
\text { food intake and } 13 \\
\text { on milk intake }\end{array}$ & $\begin{array}{c}\text { dairy product } \\
\text { consumption ano } \\
\text { BC risk }\end{array}$ \\
\hline Gandini 2000 [38] & $\begin{array}{c}14 \text { studies in Europe, } 8 \text { in } \\
\text { North America, } 3 \text { in Asia, } \\
1 \text { in Australia }\end{array}$ & 1982-1999 & $\begin{array}{c}26 \text { studies focusing } \\
\text { on vegetables (17), } \\
\text { fruits (13), } \\
\text { beta-carotene (11) } \\
\text { and vitamin C (9) }\end{array}$ & 23,038 cases in all & meta-analysis & $\begin{array}{l}5 \text { cohort studies and } \\
21 \text { case-control } \\
\text { studies }\end{array}$ & $\begin{array}{l}\text { consumption of } \\
\text { fruit and } \\
\text { vegetables, and } \\
\text { associated } \\
\text { micronutrients, } \\
\text { and risk of BC } \\
\end{array}$ \\
\hline Gissel 2008 [53] & // & up to June 2007 & 6 studies & 78,712 women & meta-analysis & $\begin{array}{l}\text { epidemiological } \\
\text { studies } \\
\text { (cross-sectional, } \\
\text { case-control, or } \\
\text { cohort) and } \\
\text { randomized } \\
\text { controlled trials } \\
\text { (RCTs) }\end{array}$ & $\begin{array}{l}\text { vitamin } \mathrm{D} \text { intake } \\
\text { and } \mathrm{BC}\end{array}$ \\
\hline
\end{tabular}


Table 1. Cont.

\begin{tabular}{|c|c|c|c|c|c|c|c|}
\hline Author_Year & $\begin{array}{c}\text { Continent or Region, } \mathrm{n} . \\
\text { of Studies }\end{array}$ & $\begin{array}{l}\text { Search Range } \\
\text { Applied }\end{array}$ & $\begin{array}{c}\text { Total } \mathrm{n} \text {. of } \\
\text { Studies Included } \\
\text { in the Review }\end{array}$ & $\begin{array}{l}\text { Total Sample Size of all } \\
\text { Studies Included in the } \\
\text { Review }\end{array}$ & Type of Study & Study Design & $\begin{array}{l}\text { Association/s } \\
\text { Examined }\end{array}$ \\
\hline Guo 2015 [19] & $\begin{array}{c}6 \text { studies in the USA, } 6 \text { in } \\
\text { Europe, } 1 \text { in North } \\
\text { America and Western } \\
\text { Europe, and } 1 \text { in Asia }\end{array}$ & up to October 2014 & $\begin{array}{l}14 \text { prospective } \\
\text { studies }\end{array}$ & $\begin{array}{c}1,588,890 \text { participants and } \\
31,552 \text { BC cases }\end{array}$ & meta-analysis & $\begin{array}{l}14 \text { cohort/nested } \\
\text { case-control studies }\end{array}$ & $\begin{array}{c}\text { red meat and } \\
\text { processed meat } \\
\text { consumption and } \\
\text { BC risk }\end{array}$ \\
\hline Hu 2012 [59] & // & $\begin{array}{c}\text { January 1982-May } \\
2011\end{array}$ & 33 studies & $/ /$ & $\begin{array}{l}\text { meta-analysis and } \\
\text { meta-regression }\end{array}$ & $\begin{array}{c}24 \text { case-control } \\
\text { studies, } 1 \text { nested } \\
\text { case-control study, } 2 \\
\text { case-cohort studies } \\
\text { and } 6 \text { cohort studies }\end{array}$ & $\begin{array}{l}\text { carotenoids and } \\
\text { BC risk }\end{array}$ \\
\hline Hui 2013 [58] & $\begin{array}{l}5 \text { studies in Europe, } 5 \text { in } \\
\text { North America ( } 4 \text { in the } \\
\text { USA) and } 2 \text { in Asia } \\
\text { (China) }\end{array}$ & up to July 2012 & $\begin{array}{l}12 \text { studies } \\
\text { included }\end{array}$ & $\begin{array}{l}9513 \text { cases and } 181,906 \\
\text { controls }\end{array}$ & $\begin{array}{l}\text { meta-analysis of } \\
\text { epidemiological } \\
\text { studies }\end{array}$ & $\begin{array}{l}6 \text { cohort studies, } 6 \\
\text { case-control studies }\end{array}$ & $\begin{array}{c}\text { flavonoids, } \\
\text { flavonoid } \\
\text { subclasses and BC } \\
\text { risk }\end{array}$ \\
\hline Larsson 2007 [56] & $\begin{array}{c}12 \text { studies in North } \\
\text { America (10 in the USA), } 7 \\
\text { in Europe, } 2 \text { in Asia, } 1 \text { in } \\
\text { South America (Uruguay) } \\
\text { and } 1 \text { in Australia }\end{array}$ & $\begin{array}{l}\text { from January } 1966 \\
\text { to November } 2006\end{array}$ & $\begin{array}{l}23 \text { studies ( } 21 \\
\text { assessing dietary } \\
\text { folate intake) }\end{array}$ & $\begin{array}{c}8367 \mathrm{BC} \text { cases out of } \\
302,959 \text { participants in } \\
\text { cohort studies; } 8558 \mathrm{BC} \\
\text { cases and } 10,812 \text { control } \\
\text { subjects in case-control } \\
\text { studies }\end{array}$ & meta-analysis & $\begin{array}{l}9 \text { cohort studies and } \\
14 \text { case-control } \\
\text { studies }\end{array}$ & $\begin{array}{c}\text { dietary folate } \\
\text { intake and BC risk }\end{array}$ \\
\hline Li 2014 [41] & $\begin{array}{c}8 \text { case-control studies in } \\
\text { Asia, } 2 \text { cohort studies in } \\
\text { Europe }\end{array}$ & up to 2013 & $\begin{array}{c}7 \text { articles including } \\
10 \text { studies }\end{array}$ & $\begin{array}{c}2313 \text { BC cases and } 2387 \\
\text { controls in case-control } \\
\text { studies; } 4577 \text { cases among } \\
\text { 1,748,623 follow-up } \\
\text { person/years }\end{array}$ & meta-analysis & $\begin{array}{l}2 \text { cohort and } 8 \\
\text { case-control studies }\end{array}$ & $\begin{array}{l}\text { dietary mushroom } \\
\text { intake and BC risk }\end{array}$ \\
\hline Mulholland 2008 & $\begin{array}{c}8 \text { studies in North-Central } \\
\text { America, } 5 \text { in Europe and } \\
1 \text { Australia }\end{array}$ & up to May 2008 & 14 studies & $\begin{array}{c}17,673 \text { BC cases out of } \\
492,011 \text { participants }\end{array}$ & $\begin{array}{l}\text { systematic review } \\
\text { and meta-analysis }\end{array}$ & $\begin{array}{c}10 \text { cohort, } 4 \\
\text { case-control studies }\end{array}$ & $\begin{array}{l}\text { dietary glycemic } \\
\text { index, glycemic } \\
\text { load and BC risk }\end{array}$ \\
\hline Mullie 2016 [48] & $\begin{array}{l}6 \text { studies in North } \\
\text { America, } 4 \text { in Europe, } 1 \text { in } \\
\text { Australia and } 1 \text { in China }\end{array}$ & $\begin{array}{l}\text { up to December } \\
2011\end{array}$ & 12 studies & $\begin{array}{c}20,973 \mathrm{BC} \text { cases out of } \\
773,971 \text { subjects }\end{array}$ & $\begin{array}{c}\text { meta-analysis of } \\
\text { prospective cohort } \\
\text { studies }\end{array}$ & 12 cohort studies & $\begin{array}{l}\text { high glycemic } \\
\text { index, glycemic } \\
\text { load and BC risk }\end{array}$ \\
\hline
\end{tabular}


Table 1. Cont.

\begin{tabular}{|c|c|c|c|c|c|c|c|}
\hline Author_Year & $\begin{array}{c}\text { Continent or Region, } \mathrm{n} \text {. } \\
\text { of Studies }\end{array}$ & $\begin{array}{l}\text { Search Range } \\
\text { Applied }\end{array}$ & $\begin{array}{c}\text { Total n. of } \\
\text { Studies Included } \\
\text { in the Review }\end{array}$ & $\begin{array}{c}\text { Total Sample Size of all } \\
\text { Studies Included in the } \\
\text { Review }\end{array}$ & Type of Study & Study Design & $\begin{array}{l}\text { Association/s } \\
\text { Examined }\end{array}$ \\
\hline $\begin{array}{l}\text { Schwingshackl } \\
2014[15]\end{array}$ & $\begin{array}{l}7 \text { studies in Europe, } 4 \text { in } \\
\text { North America }\end{array}$ & up to January 2014 & $\begin{array}{l}11 \text { studies on } B C \\
\text { risk }\end{array}$ & $\begin{array}{l}489,109 \text { participants in } \\
\text { cohort studies; } 4990 \mathrm{BC} \\
\text { cases and } 5446 \text { controls in } \\
\text { case-control studies }\end{array}$ & $\begin{array}{l}\text { systematic review } \\
\text { and meta-analysis }\end{array}$ & $\begin{array}{l}5 \text { case-control and } 6 \\
\text { cohort studies }\end{array}$ & $\begin{array}{l}\text { Mediterranean } \\
\text { diet and } B C \text { risk }\end{array}$ \\
\hline Seely 2005 [42] & $\begin{array}{c}4 \text { studies in Asian } \\
\text { populations (Japanese, } \\
\text { Chinese or Filipino) in } \\
\text { Asia, } 1 \text { in Asian } \\
\text { populations of the USA }\end{array}$ & $\begin{array}{l}\text { from } 1966 \text { to } \\
\text { November } 2004\end{array}$ & $\begin{array}{c}7 \text { studies (5 } \\
\text { prevention studies } \\
\text { examining risk of } \\
\text { primary } \mathrm{BC} \text { and } \\
\text { green tea } \\
\text { consumption) }\end{array}$ & $\begin{array}{c}857 \text { primary BC cases and } \\
1519 \text { controls in } \\
\text { case-control studies; } \\
115,601 \text { participants in } \\
\text { cohort studies }\end{array}$ & $\begin{array}{l}\text { systematic review } \\
\text { and meta-analysis }\end{array}$ & $\begin{array}{c}3 \text { cohort studies and } \\
2 \text { case-control } \\
\text { studies }\end{array}$ & $\begin{array}{c}\text { green tea } \\
\text { consumption and } \\
\text { BC risk }\end{array}$ \\
\hline Si 2014 [25] & $\begin{array}{l}5 \text { studies in Asia, } 4 \text { in } \\
\text { Europe, } 2 \text { in the USA, } 1 \text { in } \\
\text { South America and } 1 \text { in } \\
\text { both Europe and America }\end{array}$ & $\begin{array}{l}\text { up to February } \\
2013\end{array}$ & 13 studies & $\begin{array}{c}\text { 19,280 BC cases among } \\
\text { 811,555 participants in } \\
\text { cohort studies; } 13,949 \\
\text { cases and } 37,883 \text { controls } \\
\text { in case-control studies }\end{array}$ & meta-analysis & $\begin{array}{c}5 \text { cohort studies and } \\
8 \text { case-control } \\
\text { studies }\end{array}$ & $\begin{array}{l}\text { egg consumption } \\
\text { and } \mathrm{BC} \text { risk }\end{array}$ \\
\hline Song 2013 [40] & $\begin{array}{l}3 \text { studies in China, } 2 \text { in the } \\
\text { USA, } 1 \text { in Australia }\end{array}$ & up to January 2012 & 6 studies & $\begin{array}{l}8393 \text { participants: } 3789 \\
\text { cases and } 4705 \text { controls }\end{array}$ & $\begin{array}{c}\text { quantitative } \\
\text { systematic review } \\
\text { and meta-analysis }\end{array}$ & $\begin{array}{l}6 \text { case-control } \\
\text { studies }\end{array}$ & $\begin{array}{c}\text { association } \\
\text { between citrus } \\
\text { fruit intake and BC } \\
\text { risk }\end{array}$ \\
\hline Taylor 2009 [20] & $\begin{array}{c}5 \text { studies in North } \\
\text { America, } 2 \text { in Europe, } 2 \text { in } \\
\text { Asia and } 1 \text { in South } \\
\text { America }\end{array}$ & from 1966 to 2009 & 10 studies & $\begin{array}{c}1842 \text { BC cases and } 2124 \\
\text { controls in case-control } \\
\text { studies; } 2024 \text { BC cases } \\
\text { among 192,627 } \\
\text { participants in cohort } \\
\text { studies } \\
\end{array}$ & meta-analysis & $\begin{array}{c}6 \text { case-control } \\
\text { studies, } 1 \text { nested } \\
\text { case-control and } 3 \\
\text { cohort studies }\end{array}$ & $\begin{array}{l}\text { red meat intake } \\
\text { and } B C \text { risk }\end{array}$ \\
\hline Trock 2006 [31] & $\begin{array}{l}8 \text { studies in Asian } \\
\text { populations and } 10 \text { in } \\
\text { Western populations }\end{array}$ & from 1978 to 2004 & 18 studies & 9182 BC cases & meta-analysis & $\begin{array}{l}12 \text { case-control and } 6 \\
\text { cohort or nested } \\
\text { case-control studies }\end{array}$ & $\begin{array}{l}\text { soy intake and BC } \\
\text { risk }\end{array}$ \\
\hline Turner 2011 [45] & $\begin{array}{c}25 \text { studies in North } \\
\text { America (USA), } 18 \text { in } \\
\text { Europe, } 2 \text { in South } \\
\text { America (Uruguay), } 6 \text { in } \\
\text { Asia, and } 1 \text { in Australia }\end{array}$ & up to May 2010 & 52 studies & $\begin{array}{c}44,070 \mathrm{BC} \text { cases among } \\
1,659,913 \\
\text { participants/controls }\end{array}$ & meta-analysis & $\begin{array}{c}25 \text { cohort studies } \\
\text { and } 27 \text { case-control } \\
\text { studies }\end{array}$ & $\begin{array}{l}\text { fat intake and } B C \\
\text { risk }\end{array}$ \\
\hline
\end{tabular}


Table 1. Cont.

\begin{tabular}{|c|c|c|c|c|c|c|c|}
\hline Author_Year & $\begin{array}{l}\text { Continent or Region, } \\
\text { n. of Studies }\end{array}$ & $\begin{array}{l}\text { Search Range } \\
\text { Applied }\end{array}$ & $\begin{array}{l}\text { Total n. of Studies } \\
\text { Included in the } \\
\text { Review }\end{array}$ & $\begin{array}{c}\text { Total Sample Size of all } \\
\text { Studies Included in the } \\
\text { Review }\end{array}$ & Type of Study & Study Design & $\begin{array}{l}\text { Association/s } \\
\text { Examined }\end{array}$ \\
\hline $\begin{array}{c}\text { Velentzis } 2009 \\
\text { [32] }\end{array}$ & $\begin{array}{c}6 \text { studies conducted in } \\
\text { North America, } 6 \text { in } \\
\text { Europe }\end{array}$ & $\begin{array}{l}\text { up to September } \\
2008\end{array}$ & $\begin{array}{l}23 \text { studies, } 12 \text { of them } \\
\text { on plant lignan intake }\end{array}$ & $\begin{array}{l}12,430 \mathrm{BC} \text { cases among } \\
258,984 \text { participants }\end{array}$ & meta-analyses & $\begin{array}{c}5 \text { cohort and } 7 \\
\text { case-control studies }\end{array}$ & $\begin{array}{l}\text { lignans and } B C \\
\text { risk }\end{array}$ \\
\hline Wu 2008 [9] & $\begin{array}{c}8 \text { studies in Asia and } \\
\text { Asian Americans, } 11 \\
\text { in Western } \\
\text { populations }\end{array}$ & // & 19 studies & // & meta-analysis & $\begin{array}{c}14 \text { case-control } \\
\text { studies and } 5 \\
\text { cohort/nested } \\
\text { case-control studies }\end{array}$ & $\begin{array}{c}\text { soy exposure and } \\
\text { BC risk }\end{array}$ \\
\hline Wu 2015 [33] & $\begin{array}{l}\text { All studies on Chinese } \\
\text { populations }\end{array}$ & up to June 2013 & 22 studies & $\begin{array}{l}23,201 \text { subjects: } 10,566 \text { in } \\
\text { the experimental group } \\
\text { (cases) and 12,635 controls }\end{array}$ & $\begin{array}{l}\text { systematic review } \\
\text { and meta-analysis }\end{array}$ & $\begin{array}{c}21 \text { case-control } \\
\text { studies and } 1 \text { cohort } \\
\text { study }\end{array}$ & $\begin{array}{c}\text { dietary factors and } \\
\text { BC risk }\end{array}$ \\
\hline Yang 2014 [49] & $\begin{array}{c}4 \text { studies in the USA, } 5 \\
\text { in Europe and } 2 \text { in } \\
\text { Asia }\end{array}$ & up to April 2013 & 11 prospective studies & $\begin{array}{l}8331 \mathrm{BC} \text { events among } \\
274,135 \text { adult women }\end{array}$ & meta-analysis & $\begin{array}{c}6 \text { nested case-control } \\
\text { studies and } 5 \text { cohort } \\
\text { studies }\end{array}$ & $\begin{array}{l}\mathrm{n}-3 / \mathrm{n}-6 \text { PUFAs and } \\
\text { risk of } \mathrm{BC}\end{array}$ \\
\hline Zang 2015 [27] & $\begin{array}{c}11 \text { cohort studies in } \\
\text { the USA, } 10 \text { in Europe, } \\
1 \text { in Japan; } 5 \\
\text { case-control studies in } \\
\text { Asia }\end{array}$ & up to January 2014 & 27 studies & $\begin{array}{c}\text { 37,925 BC cases among } \\
1,566,940 \text { participants in } \\
\text { cohort studies and } 7418 \\
\text { incident BC cases among } \\
\text { 33,372 participants in } \\
\text { case-control studies }\end{array}$ & $\begin{array}{l}\text { systematic review } \\
\text { and meta-analysis }\end{array}$ & $\begin{array}{l}22 \text { cohort studies } \\
\text { and } 5 \text { case-control } \\
\text { studies }\end{array}$ & $\begin{array}{l}\text { dairy intake and } \\
\text { BC }\end{array}$ \\
\hline Zheng 2013 [50] & $\begin{array}{c}11 \text { studies in USA, } 11 \\
\text { in Europe and } 4 \text { in } \\
\text { Asia }\end{array}$ & $\begin{array}{l}\text { up to December } \\
2012\end{array}$ & $\begin{array}{c}26 \text { publications on } 21 \\
\text { prospective studies: } \\
\text { (14 on fish intake and } \\
19 \text { on marine } n-3 \\
\text { PUFA) }\end{array}$ & $\begin{array}{l}20,905 \mathrm{BC} \text { cases and } \\
883,585 \text { participants }\end{array}$ & meta-analysis & $\begin{array}{c}26 \text { cohort studies (8 } \\
\text { nested case-control } \\
\text { and } 2 \text { case-cohort } \\
\text { studies) }\end{array}$ & $\begin{array}{c}\text { intake of fish and } \\
\text { marine omega-3 } \\
\text { polyunsaturated } \\
\text { fatty acids (n-3 } \\
\text { PUFA)and risk of } \\
\text { BC }\end{array}$ \\
\hline Zhou 2015 [51] & $\begin{array}{c}6 \text { studies in Europe, } 5 \\
\text { in the USA and } 1 \text { in } \\
\text { Asia (China) }\end{array}$ & $\begin{array}{l}\text { up to November } \\
2014\end{array}$ & $\begin{array}{l}12 \text { studies, } 2 \text { of which } \\
\text { reported two separate } \\
\text { outcomes (pre- and } \\
\text { post-menopausal), for } \\
\text { a total of } 14 \text { outcomes } \\
\text { included in the } \\
\text { meta-analysis }\end{array}$ & $\begin{array}{l}\text { 10,410 BC events among } \\
358,955 \text { adult females }\end{array}$ & meta-analysis & $\begin{array}{l}8 \text { cohort studies and } \\
4 \text { nested case-control } \\
\text { studies }\end{array}$ & $\begin{array}{l}\text { linoleic acid and } \\
\text { BC risk }\end{array}$ \\
\hline
\end{tabular}


Table 1. Cont.

\begin{tabular}{|c|c|c|c|c|c|c|c|}
\hline Author_Year & $\begin{array}{c}\text { Continent or Region, } \mathrm{n} \text {. } \\
\text { of Studies }\end{array}$ & $\begin{array}{l}\text { Search Range } \\
\text { Applied }\end{array}$ & $\begin{array}{c}\text { Total n. of } \\
\text { Studies Included } \\
\text { in the Review }\end{array}$ & $\begin{array}{l}\text { Total Sample Size of all } \\
\text { Studies Included in the } \\
\text { Review }\end{array}$ & Type of Study & Study Design & $\begin{array}{l}\text { Association/s } \\
\text { Examined }\end{array}$ \\
\hline \multicolumn{8}{|c|}{ Characteristics of pooled analyses included in the umbrella review (4) } \\
\hline $\begin{array}{c}\text { Mannisto } 2005 \\
{[16]}\end{array}$ & & // & 3 cohort studies & $\begin{array}{c}\text { 77,037 participants and } \\
3271 \text { BC cases }\end{array}$ & pooled analysis & $\begin{array}{l}3 \text { cohort studies in the } \\
\text { DIETSCAN project: } \\
\text { Netherlands Cohort } \\
\text { Study on diet and } \\
\text { cancer (NCLS), the } \\
\text { Italian "Ormoni e } \\
\text { Dieta nella Eziologia } \\
\text { dei Tumori"(ORDET) } \\
\text { and the Swedish } \\
\text { Mammography } \\
\text { Cohort (SMC) }\end{array}$ & $\begin{array}{l}\text { dietary pattern } \\
\text { and BC risk }\end{array}$ \\
\hline Missmer 2002 [22] & $\begin{array}{l}\text { North America and } \\
\text { Western Europe }\end{array}$ & // & 8 studies & $\begin{array}{l}7379 \text { BC cases out of } \\
351,041 \text { women }\end{array}$ & pooled analysis & 8 cohort studies & $\begin{array}{l}\text { meat and dairy } \\
\text { food consumption } \\
\text { and risk of } B C\end{array}$ \\
\hline $\begin{array}{l}\text { Smith-Warner } \\
2001 \text { (a) [46] }\end{array}$ & $\begin{array}{l}7 \text { studies in North } \\
\text { America, } 2 \text { in Europe }\end{array}$ & up to 2001 & $\begin{array}{l}8 \text { studies ( } 9 \text { in all, } \\
\text { because the } \\
\text { Nurse's Health } \\
\text { Study was divided } \\
\text { in two) }\end{array}$ & $\begin{array}{l}7329 \text { BC cases out of } \\
351,821 \text { participants }\end{array}$ & $\begin{array}{l}\text { pooled analysis of } \\
\text { cohort studies }\end{array}$ & $\begin{array}{c}\text { pooling project: } 9 \\
\text { prospective studies (8 } \\
\text { analyzed as nested } \\
\text { case-control studies, } 1 \\
\text { used a case-cohort } \\
\text { design) }\end{array}$ & $\begin{array}{l}\text { types of dietary fat } \\
\text { and BC risk }\end{array}$ \\
\hline $\begin{array}{l}\text { Smith-Warner } \\
2001 \text { (b) [39] }\end{array}$ & $\begin{array}{l}7 \text { studies in North } \\
\text { America, } 2 \text { in Europe }\end{array}$ & up to 2001 & $\begin{array}{l}8 \text { studies ( } 9 \text { in all, } \\
\text { because the } \\
\text { Nurse's Health } \\
\text { Study was divided } \\
\text { in two) }\end{array}$ & $\begin{array}{c}7377 \text { incident } B C \text { cases } \\
\text { among } 351,825 \\
\text { participants }\end{array}$ & $\begin{array}{l}\text { pooled analysis of } \\
\text { cohort studies }\end{array}$ & $\begin{array}{c}\text { pooling project: } 9 \\
\text { prospective studies (8 } \\
\text { analyzed as nested } \\
\text { case-control studies, } 1 \\
\text { used a case-cohort } \\
\text { design) }\end{array}$ & $\begin{array}{l}\text { intake of fruits and } \\
\text { vegetables and BC } \\
\text { risk }\end{array}$ \\
\hline \multicolumn{8}{|c|}{ Characteristics of systematic reviews included in the umbrella review (5) } \\
\hline $\begin{array}{l}\text { Albuquerque } \\
2013[17]\end{array}$ & $\begin{array}{l}8 \text { studies in Europe, } 9 \text { in } \\
\text { North America, } 5 \text { in Asia, } \\
4 \text { in South America, } 1 \text { in } \\
\text { Africa, and } 1 \text { in Oceania }\end{array}$ & $\begin{array}{l}\text { up December } \\
2012\end{array}$ & 26 articles & $\begin{array}{l}584,437 \text { women and } 28,962 \\
\text { incident cases of BC }\end{array}$ & systematic review & $\begin{array}{l}11 \text { cohort studies and } \\
15 \text { case-control studies }\end{array}$ & $\begin{array}{l}\text { dietary patterns } \\
\text { and } B C \text { risk }\end{array}$ \\
\hline
\end{tabular}


Table 1. Cont

\begin{tabular}{|c|c|c|c|c|c|c|c|}
\hline Author_Year & $\begin{array}{c}\text { Continent or Region, } n \text {. } \\
\text { of Studies }\end{array}$ & $\begin{array}{l}\text { Search Range } \\
\text { Applied }\end{array}$ & $\begin{array}{l}\text { Total } \mathbf{n} \text {. of } \\
\text { Studies Included } \\
\text { in the Review }\end{array}$ & $\begin{array}{l}\text { Total Sample Size of all } \\
\text { Studies Included in the } \\
\text { Review }\end{array}$ & Type of Study & Study Design & $\begin{array}{l}\text { Association } / \mathrm{s} \\
\text { Examined }\end{array}$ \\
\hline $\begin{array}{c}\text { Farsinejad-Marj } \\
\quad 2015[18]\end{array}$ & $\begin{array}{c}7 \text { studies in Europe, } 1 \text { in } \\
\text { America }\end{array}$ & up to April 2015 & 8 studies & 502,253 participants & systematic review & $\begin{array}{l}5 \text { cohort studies; } 3 \\
\text { case-control studies }\end{array}$ & $\begin{array}{l}\text { Mediterranean } \\
\text { diet and } B C \text { risk }\end{array}$ \\
\hline Michels 2007 [28] & not clearly defined & $\begin{array}{c}\text { January 1950-May } \\
2005\end{array}$ & $\begin{array}{c}21 \text { studies on fat } \\
\text { intake, } 8 \text { on fruit } \\
\text { and vegetables, } 11 \\
\text { on carbohydrates, } \\
11 \text { on antioxidants, } \\
12 \text { on dairy } \\
\text { products, } 2 \text { on } \\
\text { vitamin } D, 5 \text { on } \\
\text { soy, } 6 \text { on green tea, } \\
1 \text { on heterocyclic } \\
\text { amines, } 2 \text { on } \\
\text { adolescent diet }\end{array}$ & not clearly defined & systematic review & $\begin{array}{l}73 \text { cohort studies, } 3 \\
\text { meta-analyses, } 3 \\
\text { pooled analyses }\end{array}$ & diet and $B C$ \\
\hline $\begin{array}{c}\text { Mourouti } 2013 \\
{[34]}\end{array}$ & $/ /$ & $\begin{array}{c}\text { from January } 2000 \\
\text { to April } 2013\end{array}$ & $\begin{array}{l}29 \text { studies, } 12 \text { on } \\
\text { populations with a } \\
\text { high consumption } \\
\text { of soy }\end{array}$ & // & systematic review & $\begin{array}{l}10 \text { cohort, } 3 \text { nested } \\
\text { case-control and } 16 \\
\text { case-control studies }\end{array}$ & $\begin{array}{c}\text { soy food } \\
\text { consumption and } \\
\text { BC risk }\end{array}$ \\
\hline $\begin{array}{c}\text { Mourouti } 2015 \\
{[21]}\end{array}$ & // & $\begin{array}{c}\text { from January } 2002 \\
\text { to August } 2012\end{array}$ & $\begin{array}{c}95 \text { studies on: fruit } \\
\text { and vegetable (11), } \\
\text { meat (14), soy (24), } \\
\text { dietary fiber (9), } \\
\text { dietary } \\
\text { carbohydrates (16), } \\
\text { dietary lipids (21) }\end{array}$ & $\begin{array}{c}\text { fruit and veg: } 16,110 \text { cases } \\
\text { among } 122,933 \text { participants } \\
\text { meat: } 31,918 \text { cases among } \\
813.021 \text { participants soy: } \\
9064 \text { cases among } 324,345 \\
\text { participants dietary fiber: } \\
\text { 15,880 cases among } 641,322 \\
\text { participants fat: } 24,788 \text { cases } \\
\text { among } 951,479 \text { participants } \\
\text { diet carbohydrates (studies } \\
\text { showing a positive } \\
\text { association): } 4332 \text { cases } \\
\text { among } 146,692 \text { participants }\end{array}$ & systematic review & $\begin{array}{l}42 \text { case-control } \\
\text { studies, } 51 \text { cohort } \\
\text { studies and } 2 \text { dietary } \\
\text { intervention RCTs }\end{array}$ & diet and $B C$ \\
\hline
\end{tabular}


Table 1. Cont.

\begin{tabular}{|c|c|c|c|c|c|c|c|}
\hline Author_Year & $\begin{array}{c}\text { Continent or Region, } n \text {. } \\
\text { of Studies }\end{array}$ & $\begin{array}{l}\text { Search Range } \\
\text { Applied }\end{array}$ & $\begin{array}{c}\text { Total n. of } \\
\text { Studies Included } \\
\text { in the Review }\end{array}$ & $\begin{array}{l}\text { Total Sample Size of all } \\
\text { Studies Included in the } \\
\text { Review }\end{array}$ & Type of Study & Study Design & $\begin{array}{l}\text { Association } / \mathrm{s} \\
\text { Examined }\end{array}$ \\
\hline \multicolumn{8}{|c|}{ Characteristics of not systematic reviews included in the umbrella review (7) } \\
\hline Cui 2006 [54] & $\begin{array}{c}\text { vitamin D: } 7 \text { studies in } \\
\text { North America (6 in USA), } \\
1 \text { in Europe (Switzerland) } \\
\text { Calcium: } 9 \text { studies in } \\
\text { Europe, } 2 \text { in North } \\
\text { America, and } 1 \text { in Asia } \\
\text { (China) }\end{array}$ & not defined & $\begin{array}{c}20 \text { studies ( } 8 \text { on } \\
\text { dietary vitamin } \mathrm{D} \\
\text { and } 12 \text { on calcium } \\
\text { intake) }\end{array}$ & $\begin{array}{l}\text { vitamin D: } 7947 \text { BC cases } \\
\text { out of } 218,776 \text { subjects; } \\
\text { calcium: } 11,378 \text { BC cases } \\
\text { out of } 16,764 \text { subjects }\end{array}$ & $\begin{array}{l}\text { not-systematic } \\
\text { review }\end{array}$ & $\begin{array}{c}\text { vitamin D: } 5 \\
\text { prospective studies } \\
(4 \text { cohort and } 1 \\
\text { nested case-control) } \\
\text { and } 3 \text { case control } \\
\text { studies; } \\
\text { calcium: } 3 \text { cohort } \\
\text { and } 9 \text { case-control } \\
\text { studies }\end{array}$ & $\begin{array}{c}\text { association } \\
\text { between vitamin D } \\
\text { and calcium and } \\
\text { BC risk }\end{array}$ \\
\hline Duffy 2007 [35] & $\begin{array}{l}5 \text { studies in Europe, } 8 \text { in } \\
\text { Asia, } 6 \text { North America }\end{array}$ & // & 21 studies & $\begin{array}{c}12,472 \text { BC cases and } \\
35,513 \text { controls in } \\
\text { case-control studies; } 1799 \\
\text { BC cases among 206,030 } \\
\text { participants in cohort } \\
\text { studies }\end{array}$ & $\begin{array}{l}\text { not-systematic } \\
\text { review }\end{array}$ & $\begin{array}{c}6 \text { cohort studies and } \\
15 \text { case-control } \\
\text { studies }\end{array}$ & $\begin{array}{c}\text { phytoestrogen } \\
\text { intake and BC risk }\end{array}$ \\
\hline $\begin{array}{c}\text { Eichholzer } 2001 \\
{[57]}\end{array}$ & $\begin{array}{c}6 \text { studies in North } \\
\text { America, } 1 \text { in South } \\
\text { America, and } 1 \text { in Europe }\end{array}$ & // & 8 studies & $9420 \mathrm{BC}$ cases & $\begin{array}{l}\text { not-systematic } \\
\text { review }\end{array}$ & $\begin{array}{c}5 \text { case control } \\
\text { studies and } 3 \text { nested } \\
\text { case-control studies }\end{array}$ & $\begin{array}{l}\text { folate intake and } \\
\text { BC risk }\end{array}$ \\
\hline Hanf 2005 [23] & // & // & $\begin{array}{l}13 \text { prospective } \\
\text { studies on fat } \\
\text { intake; } 10 \\
\text { prospective } \\
\text { studies on meat } \\
\text { consumption }\end{array}$ & // & $\begin{array}{l}\text { not-systematic } \\
\text { review }\end{array}$ & $\begin{array}{l}\text { cohort studies and } \\
\text { interventional trials }\end{array}$ & $\begin{array}{l}\text { foods, nutrients } \\
\text { and } \mathrm{BC} \text { risk }\end{array}$ \\
\hline Lof $2006[36]$ & $\begin{array}{c}5 \text { studies in North } \\
\text { America, } 3 \text { in Europe ( } 1 \text { of } \\
\text { them on an Asian } \\
\text { population) }\end{array}$ & $\begin{array}{l}\text { from } 1966 \text { to } \\
\text { September } 2006\end{array}$ & 7 studies & $\begin{array}{c}4741 \mathrm{BC} \text { cases among } \\
134,100 \text { participants }\end{array}$ & $\begin{array}{l}\text { not-systematic } \\
\text { review }\end{array}$ & $\begin{array}{c}5 \text { case-control } \\
\text { studies and } 2 \text { cohort } \\
\text { studies }\end{array}$ & $\begin{array}{c}\text { dietary } \\
\text { phytoestrogens } \\
\text { intake and BC risk }\end{array}$ \\
\hline Peeters 2003 [37] & $\begin{array}{c}2 \text { studies in North } \\
\text { America and } 11 \text { on Asian } \\
\text { populations }\end{array}$ & $\begin{array}{l}\text { up to November } \\
2011\end{array}$ & $\begin{array}{l}13 \text { studies on } \\
\text { dietary } \\
\text { phytoestrogens }\end{array}$ & $\begin{array}{l}5954 \text { BC cases out of } \\
27,162 \text { participants }\end{array}$ & $\begin{array}{l}\text { not-systematic } \\
\text { review }\end{array}$ & $\begin{array}{c}9 \text { case-control } \\
\text { studies and } 4 \text { cohort } \\
\text { studies }\end{array}$ & $\begin{array}{l}\text { phytoestrogens } \\
\text { and } \mathrm{BC} \text { risk }\end{array}$ \\
\hline
\end{tabular}


Table 1. Cont

\begin{tabular}{|c|c|c|c|c|c|c|c|}
\hline Author_Year & $\begin{array}{c}\text { Continent or Region, } n \text {. } \\
\text { of Studies }\end{array}$ & $\begin{array}{l}\text { Search Range } \\
\text { Applied }\end{array}$ & $\begin{array}{l}\text { Total n. of Studies } \\
\text { Included in the } \\
\text { Review }\end{array}$ & $\begin{array}{l}\text { Total Sample Size of all } \\
\text { Studies Included in the } \\
\text { Review }\end{array}$ & Type of Study & Study Design & $\begin{array}{l}\text { Association/s } \\
\text { Examined }\end{array}$ \\
\hline Rossi 2014 [60] & // & 1975-2013 & $\begin{array}{l}175 \text { pertinent articles: } \\
18 \text { for proteins, } 18 \text { for } \\
\text { carbohydrates, } 18 \text { for } \\
\text { dietary fat, } 33 \text { for } \\
\text { polyphenols and } \\
\text { phytoestrogen, } 18 \text { for } \\
\text { fruit and vegetables, } \\
11 \text { for lycopene, } 44 \text { for } \\
\text { vitamins and oligo } \\
\text { elements, } 8 \text { for alcohol }\end{array}$ & // & $\begin{array}{l}\text { not-systematic } \\
\text { review }\end{array}$ & // & $\begin{array}{l}\text { impact of dietary } \\
\text { factors in } B C \text { risk }\end{array}$ \\
\hline
\end{tabular}

Table 2. Results of studies included in the umbrella review.

\begin{tabular}{|c|c|c|c|c|c|c|}
\hline Author_Year & Menopausal Status & Exposure Measure & Overall Results of Review & Statistical Method & $\begin{array}{l}\text { Summary Estimates and Related } \\
\qquad 95 \% \text { CI }\end{array}$ & $p$-value \\
\hline \multicolumn{7}{|c|}{ Results of meta-analysis included in the umbrella review (32) } \\
\hline Alexander 2010 [43] & $\begin{array}{c}\text { pre- and } \\
\text { post-menopause }\end{array}$ & $\begin{array}{c}\text { animal fat intake } \\
\text { (highest vs. lowest } \\
\text { categories) }\end{array}$ & $\begin{array}{c}\text { no statistically significant } \\
\text { association was found between } \\
\text { animal fat intake (comparing } \\
\text { highest vs. lowest category) and } \\
\text { BC risk. }\end{array}$ & random effect model & $\begin{array}{l}\text { summary relative risk (RR) } \\
\text { estimates: } 1.11(0.91-1.36)\end{array}$ & // \\
\hline Aune 2012 [38] & $\begin{array}{c}\text { pre- and } \\
\text { post-menopause }\end{array}$ & $\begin{array}{l}\text { dietary fiber, fruit } \\
\text { fiber, vegetable fiber, } \\
\text { cereal fiber, soluble } \\
\text { and insoluble fiber }\end{array}$ & $\begin{array}{l}\text { an inverse association was found } \\
\text { between dietary fiber intake and } \\
\text { BC risk; the association appeared } \\
\text { to be most pronounced in studies } \\
\text { with high levels ( }>25 \text { vs. } \\
<25 \text { g/day) or large ranges ( }>13 \text { vs. } \\
<13 \text { g/day) of fiber intake. }\end{array}$ & $\begin{array}{c}\text { random effects model } \\
\text { was used to calculate } \\
\text { summary RRs and } \\
95 \% \text { CIs }\end{array}$ & $\begin{array}{c}\text { summary RR (high vs. low intake): } \\
\text { - dietary fiber } 0.93(0.89-0.98) \\
\text { - fruit fiber } 0.95(0.86-1.06) \\
\text { - vegetable fiber } 0.99(0.92-1.07) \\
\text { - cereal fiber } 0.96(0.90-1.02) \\
\text { - soluble and insoluble fiber } 0.91 \\
(0.84-0.99)\end{array}$ & // \\
\hline
\end{tabular}


Table 2. Cont

\begin{tabular}{|c|c|c|c|c|c|c|}
\hline Author_Year & $\begin{array}{c}\text { Menopausal } \\
\text { Status }\end{array}$ & Exposure Measure & Overall Results of Review & Statistical Method & $\begin{array}{l}\text { Summary Estimates and Related } \\
\qquad 95 \% \text { CI }\end{array}$ & $p$-value \\
\hline Boyd 2003 [44] & // & $\begin{array}{l}\text { dietary fat: total fat, } \\
\text { saturated fat and } \\
\text { monounsaturated fat; } \\
\text { foods containing } \\
\text { animal fat: meat, milk } \\
\text { and cheese }\end{array}$ & $\begin{array}{l}\text { a higher fat intake is associated with a } \\
\text { higher risk of BC, with a significant positive } \\
\text { association confirmed for total fat and } \\
\text { saturated fat; meat and cheese consumption } \\
\text { was also associated with a statistically } \\
\text { significant increase in BC risk. }\end{array}$ & random effects model & $\begin{array}{c}\text { summary RR (highest vs. lowest } \\
\text { categories of intake) for all studies } \\
\text { combined } \\
\text { - total fat } 1.13(1.03-1.25) \\
\text { - saturated fat } 1.19(1.06-1.35) \\
\text { - monounsaturated fat } 1.11(0.96-1.28) \\
\text { - polyunsaturated fat } 0.94(0.80-1.10) \\
\text { - meat consumption } 1.17(1.06-1.29) \\
\text { - milk consumption } 1.12(0.88-1.43) \\
\text { - cheese consumption 1.26 (0.96-1.66) }\end{array}$ & // \\
\hline Brennan 2010 [14] & // & $\begin{array}{l}\text { dietary patterns (DP): } \\
\text { "prudent/healthy" vs. } \\
\text { "Western/unhealthy" }\end{array}$ & $\begin{array}{l}\text { no overall association was found for } \\
\text { Western/unhealthy DP and BC risk; results } \\
\text { of combined case-control studies showed } \\
\text { that Western DP was positively associated } \\
\text { with BC risk (highest vs. lowest category of } \\
\text { intake); there was evidence of a lower risk of } \\
\text { BC (highest vs. lowest category of intake) } \\
\text { associated with a prudent/healthy DP in all } \\
\text { studies and in pooled cohort studies. }\end{array}$ & random effects model & $\begin{array}{c}\text { summary RR estimates (highest vs. } \\
\text { lowest categories of intake): } \\
\text { Western/unhealthy diet overall 1.09 } \\
(0.98-1.22) ; \\
\text { - case-control combined 1.31 (1.04-1.63); } \\
\text { - cohort combined 0.99 (0.90-1.08) } \\
\text { Prudent/healthy diet overall 0.89 } \\
(0.82-0.99) ; \\
\text { - case-control combined } 0.84(0.67-1.04) ; \\
\text { - cohort combined } 0.93(0.88-0.98)\end{array}$ & $\begin{array}{l}0.11 \\
0.02 \\
0.82 \\
0.02 \\
0.12 \\
0.01\end{array}$ \\
\hline Buck 2010 [29] & $\begin{array}{c}\text { pre- and } \\
\text { post-menopause }\end{array}$ & $\begin{array}{l}\text { lignan exposure, } \\
\text { plant lignan intake }\end{array}$ & $\begin{array}{c}\text { lignan exposure was not associated with a } \\
\text { significantly lower risk of BC; but a high } \\
\text { lignan intake in postmenopausal women } \\
\text { was associated with a significant reduction } \\
\text { in risk of BC. }\end{array}$ & $\begin{array}{c}\text { random effects model } \\
\text { and fixed effects } \\
\text { model }\end{array}$ & $\begin{array}{l}\text { pooled estimate (highest vs. lowest } \\
\text { quantile) } \\
\text { overall } 0.92(0.81-1.02) \\
\text { - premenopause } 0.87(0.66-1.08) \\
\text { - postmenopause } 0.86(0.78-0.94) \\
\text { dietary plant lignans } 0.94(0.82-1.05) \\
\text { - premenopause } 1.01(0.87-1.15) \\
\text { - postmenopause } 0.86(0.77-0.94)\end{array}$ & // \\
\hline Chen 2010 [52] & $\begin{array}{c}\text { pre- and } \\
\text { post-menopause }\end{array}$ & $\begin{array}{l}\text { dietary calcium and } \\
\text { vitamin D intake }\end{array}$ & $\begin{array}{l}\text { a statistically significant overall inverse } \\
\text { association was found between dietary } \\
\text { vitamin D intake and BC risk; high vitamin } \\
\text { D intake was associated with a } 17 \% \text { decrease } \\
\text { in BC risk in premenopausal women; a } \\
\text { statistically significant overall inverse } \\
\text { association was found between dietary } \\
\text { calcium intake and BC risk. }\end{array}$ & $\begin{array}{l}\text { a random effects } \\
\text { model if there was } \\
\text { significant } \\
\text { heterogeneity among } \\
\text { studies; if not, a fixed } \\
\text { effects model was } \\
\text { acceptable }\end{array}$ & $\begin{array}{c}\text { summary RRs (highest vs. lowest } \\
\text { category): } \\
\text { - vitamin D intake overall } 0.91 \\
(0.83-1.00) \\
\text { premenopause } 0.83(0.73-0.95) \\
\text { postmenopause } 0.94(0.83-1.07) \\
\text { - calcium intake overall } 0.81(0.72-0.90) \\
\text { premenopause } 0.72(0.55-0.95) \\
\text { postmenopause } 0.95(0.79-1.14)\end{array}$ & // \\
\hline
\end{tabular}


Table 2. Cont

\begin{tabular}{|c|c|c|c|c|c|c|}
\hline Author_Year & Menopausal Status & Exposure Measure & Overall Results of Review & Statistical Method & $\begin{array}{l}\text { Summary Estimates and Related } \\
\qquad 95 \% \mathrm{CI}\end{array}$ & $p$-value \\
\hline Chen 2014 [55] & $\begin{array}{c}\text { pre- and } \\
\text { post-menopause }\end{array}$ & $\begin{array}{l}\text { total folate intake, } \\
\text { dietary folate intake } \\
\text { and folate } \\
\text { supplement intake }\end{array}$ & $\begin{array}{c}\text { no significant association between } \\
\text { dietary folate intake and BC risk in } \\
\text { meta-analysis of prospective } \\
\text { studies, but a significantly negative } \\
\text { correlation emerged from the case } \\
\text { control studies, particularly for } \\
\text { postmenopausal women; more } \\
\text { dietary folate may reduce the BC } \\
\text { risk in populations of Europe, } \\
\text { Australia or Asia (not in the USA). }\end{array}$ & $\begin{array}{l}\text { inverse variance } \\
\text { weighting method; } \\
\text { random effects model }\end{array}$ & $\begin{array}{c}\text { summary RRs (highest vs. lowest } \\
\text { category) in prospective studies: } \\
\text { total folate } 0.97(0.87-1.08) ; \\
\text { dietary folate (DF) } 0.95(0.87-1.03) \\
\text { - DF premenopause } 1.02(0.62-1.67) \\
\text { - DF postmenopause } 0.94(0.81-1.08) \\
\text { OR (highest vs. lowest category) in } \\
\text { case-control studies: } \\
\text { total folate } 0.87(0.61-1.23) \\
\text { dietary folate (DF) } 0.79(0.67-0.92) \\
\text { DF premenopause } 0.78(0.53-1.14) \\
\text { DF postmenopause } \mathbf{0 . 7 3}(0.59-0.92)\end{array}$ & // \\
\hline Dong 2011 (a) [30] & $\begin{array}{c}\text { pre- and } \\
\text { post-menopause }\end{array}$ & $\begin{array}{l}\text { dietary soy isoflavone } \\
\text { intake }\end{array}$ & $\begin{array}{l}\text { soy isoflavone intake was } \\
\text { associated with a significantly } \\
\text { reduced risk of BC in Asian } \\
\text { populations, but not in Western } \\
\text { populations; a significant inverse } \\
\text { association between soy isoflavone } \\
\text { intake and risk of BC emerged in } \\
\text { postmenopausal but not in } \\
\text { premenopausal women. }\end{array}$ & $\begin{array}{l}\text { random or fixed } \\
\text { effects model }\end{array}$ & $\begin{array}{c}\text { all women } 0.89(0.79-0.99) \\
\text { Asian countries } 0.76(0.65-0.86) \\
\text { Western countries } 0.97(0.87-1.06) \\
\text { premenopause } 0.90(0.64-1.15) \\
\text { postmenopause } 0.78(0.63-0.93)\end{array}$ & // \\
\hline Dong 2011 (b) [26] & $\begin{array}{c}\text { pre- and } \\
\text { post-menopause }\end{array}$ & $\begin{array}{l}\text { total dairy food } \\
\text { defined as } \\
\text { skimmed/low-fat } \\
\text { milk, whole/high-fat } \\
\text { milk, yogurt, cottage } \\
\text { cheese, butter, and } \\
\text { other dairy products }\end{array}$ & $\begin{array}{l}\text { for total dairy food consumption, a } \\
\text { significantly inverse association } \\
\text { with BC risk was confirmed, and } \\
\text { was stronger for low-fat dairy food } \\
\text { and for premenopausal women; } \\
\text { for milk consumption only low-fat } \\
\text { milk was significantly associated } \\
\text { with a lower BC risk; no } \\
\text { association in postmenopausal } \\
\text { women. }\end{array}$ & random effects model & $\begin{array}{c}\text { summary RR (highest vs. lowest } \\
\text { intake) } \\
\text { total dairy food } 0.85(0.76-0.95) \\
\text { milk } 0.90(0.80-1.02) \\
\text { total dairy food } \\
\text { - premenopause } 0.79(0.63) \\
\text { - postmenopause } 0.92(0.83-1.01) \\
\text { total milk } \\
\text { - premenopause } 0.79(0.60-1.02) \\
\text { - postmenopause } 1.01(0.94-1.09)\end{array}$ & // \\
\hline
\end{tabular}


Table 2. Cont.

\begin{tabular}{|c|c|c|c|c|c|c|}
\hline Author_Year & Menopausal Status & Exposure Measure & Overall Results of Review & Statistical Method & $\begin{array}{l}\text { Summary Estimates and Related } \\
\qquad 95 \% \text { CI }\end{array}$ & $p$-value \\
\hline Gandini 2000 [38] & // & $\begin{array}{c}\text { vegetable and fruit } \\
\text { consumption, and } 2 \\
\text { related } \\
\text { micronutrients: } \\
\text { vitamin } C \text { and } \\
\text { beta-carotene }\end{array}$ & $\begin{array}{l}\text { high vegetable consumption, and } \\
\text { high vitamin } C \text { and beta-carotene } \\
\text { intake had a significant protective } \\
\text { effect against } B C \text {; pooled estimates } \\
\text { for fruit intake indicated that this } \\
\text { food group may have a protective } \\
\text { effect, but without statistical } \\
\text { significance. }\end{array}$ & $\begin{array}{l}\text { random effects model } \\
\text { fixed effects model }\end{array}$ & $\begin{array}{l}\text { summary RR (high vs. low } \\
\text { consumption) } \\
\text { - vegetables } 0.75(0.66-0.85) \\
\text { - fruits } 0.94(0.79-1.11) \\
\text { - vitamin C } 0.80(0.68-0.95) \\
\text { - beta-carotene } 0.82(0.76-0.91)\end{array}$ & // \\
\hline Gissel 2008 [53] & $\begin{array}{c}\text { pre- and } \\
\text { post-menopause }\end{array}$ & $\begin{array}{l}\text { vitamin D } \\
\text { consumption }\end{array}$ & $\begin{array}{c}\text { no association between vitamin } \mathrm{D} \\
\text { levels and } \mathrm{BC} \text { risk. }\end{array}$ & random effects model & $\begin{array}{c}\text { summary RR: } 0.98(0.93-1.03) \\
\text { analysis restricted to intake }<400 \\
\text { International Units (IU)/day vs. } \\
\text { lower intake: } \\
\text { RR } 0.92(0.87-0.97)\end{array}$ & // \\
\hline Guo 2015 [19] & $\begin{array}{c}\text { pre- and } \\
\text { post-menopause }\end{array}$ & $\begin{array}{l}\text { red and processed } \\
\text { meat consumption }\end{array}$ & $\begin{array}{l}\text { higher red and processed meat } \\
\text { intake was associated with higher } \\
\text { risk of BC. }\end{array}$ & random effects model & $\begin{array}{c}\text { summary RR of BC (highest vs. } \\
\text { lowest consumer categories) were: } \\
1.10 \text { ( } 95 \% \text { confidence interval }(\mathrm{CI}) \\
1.02-1.19) \text { for red meat, and } 1.08 \\
(95 \% \mathrm{CI}, 1.01-1.15) \text { for processed } \\
\text { meat intake; estimated summary RR } \\
\text { were } 1.11 \text { (1.05-1.16) for an increase } \\
\text { of } 120 \mathrm{~g} / \text { day of red meat, and } 1.09 \\
(1.03-1.16) \text { for an increase of } 50 \mathrm{~g} / \text { day } \\
\text { of processed meat }\end{array}$ & $/ /$ \\
\hline Hu 2012 [59] & $\begin{array}{c}\text { pre- and } \\
\text { post-menopause }\end{array}$ & $\begin{array}{c}\text { dietary } \alpha \text {-carotene; } \\
\text { dietary } \beta \text {-carotene; } \\
\beta \text {-cryptoxanthin; } \\
\text { lutein+ zeaxanthin; } \\
\text { lycopene }\end{array}$ & $\begin{array}{l}\text { a significant inverse association } \\
\text { between } \alpha \text {-carotene consumption } \\
\text { and } \mathrm{BC} \text { risk, in both cohort and } \\
\text { case-control studies; a trend towards } \\
\text { a protective effect of } \beta \text {-carotene; no } \\
\text { significant association between } \\
\beta \text {-cryptoxanthin, lutein+zeaxanthin } \\
\text { and BC risk; lycopene may have a } \\
\text { protective effect against BC } \\
\text { (significant inverse association in } \\
\text { case-control studies). }\end{array}$ & random effects model & $\begin{array}{c}\text { summary RR among cohort studies: } \\
\alpha \text {-carotene } 0.91(0.85-0.98) \\
\beta \text {-carotene } 0.94(0.88-1.00) \\
\beta \text {-cryptoxanthin } 1.03(0.96-1.11) \\
\text { lutein+zeaxanthin } 0.94(0.87-1.02) \\
\text { lycopene } 0.99(0.93-1.06) \\
\text { summary OR among case-control } \\
\text { studies: } \\
\alpha \text {-carotene } 0.82(0.70-0.97) \\
\beta \text {-carotene } 0.75(0.67-0.85) \\
\beta \text {-cryptoxanthin } 0.95(0.80-1.13) \\
\text { lutein+zeaxanthin } 0.79(0.66-0.94) \\
\text { lycopene } 0.71(0.56-0.92)\end{array}$ & $\begin{array}{c}0.01 \\
0.05 \\
0.39 \\
0.13 \\
0.77 \\
\\
\\
0.02 \\
<0.01 \\
0.54 \\
0.01 \\
0.01\end{array}$ \\
\hline
\end{tabular}


Table 2. Cont

\begin{tabular}{|c|c|c|c|c|c|c|}
\hline Author_Year & Menopausal Status & Exposure Measure & Overall Results of Review & Statistical Method & $\begin{array}{c}\text { Summary Estimates and } \\
\text { Related } 95 \% \text { CI }\end{array}$ & $p$-value \\
\hline Hui 2013 [58] & $\begin{array}{c}\text { pre- and } \\
\text { post-menopause }\end{array}$ & $\begin{array}{c}\text { flavonoid subclasses: } \\
\text { flavonols (onions, broccoli, } \\
\text { tea, fruit); flavones (herbs, } \\
\text { parsley, chamomile); } \\
\text { flavonones (citrus fruits, } \\
\text { oranges, grapefruit); } \\
\text { flavan-3-ols (dark chocolate, } \\
\text { apples, grapes, red wine, } \\
\text { green tea); anthocyanidins } \\
\text { (berries, cranberries, black } \\
\text { currants, blueberries); } \\
\text { isoflavones (soy and soy } \\
\text { products) }\end{array}$ & $\begin{array}{l}\text { BC risk significantly lower in women } \\
\text { with high intake of flavonols and } \\
\text { flavones; no significant association } \\
\text { with flavan-3-ols, flavonones, } \\
\text { anthocyanins or total flavonoids. } \\
\text { Summary RRs of studies stratified by } \\
\text { menopausal status suggest that } \\
\text { flavonols, flavones and flavan-3-ols are } \\
\text { associated with a significantly lower } \\
\text { risk of BC in postmenopausal, but not } \\
\text { in premenopausal women. }\end{array}$ & $\begin{array}{l}\text { random and fixed } \\
\text { effects model }\end{array}$ & $\begin{array}{l}\text { summary RR (highest vs. lowest } \\
\text { consumption): } \\
\text { flavonols } 0.88(0.80-0.96) \\
\text { - postmenopause } 0.92(0.85-0.99) \\
\text { flavones } 0.83(0.76-0.91) \\
\text { - postmenopause } 0.86(0.77-0.94) \\
\text { flavan-3-ols } 0.93(0.84-1.02) \\
\text { - postmenopause } 0.90(0.83-0.98) \\
\text { flavonones } 0.95(0.88-1.03) \\
\text { anthocyanidins } 0.97(0.87-1.08) \\
\end{array}$ & $/ /$ \\
\hline Larsson $2007[56]$ & $\begin{array}{c}\text { pre- and } \\
\text { post-menopause }\end{array}$ & dietary folate intake & $\begin{array}{l}\text { no significant association between } \\
\text { dietary folate intake and BC risk in } \\
\text { prospective studies; in case-control } \\
\text { studies, high vs. low dietary folate } \\
\text { intake was associated with a } \\
\text { significantly different risk of BC (lower } \\
\text { in the former). }\end{array}$ & random effects model & $\begin{array}{l}\text { summary estimates (highest vs. } \\
\text { lowest dietary folate intake } \\
\text { categories): } \\
\text { cohort studies: RR } 0.96 \\
\text { (0.87-1.05) } \\
\text { case-control studies: Odds Ratio } \\
\text { (OR) } 0.73 \text { (0.64-0.83) }\end{array}$ & $/ /$ \\
\hline Li 2014 [41] & $\begin{array}{c}\text { pre- and } \\
\text { post-menopause }\end{array}$ & dietary mushroom intake & $\begin{array}{c}\text { a significant inverse association } \\
\text { between mushroom consumption and } \\
\text { BC risk }\end{array}$ & $\begin{array}{l}\text { random and fixed } \\
\text { effects model }\end{array}$ & $\begin{array}{c}\text { summary RR (highest vs. lowest } \\
\text { dietary mushroom intake } \\
\text { categories): } \\
\text { premenopause } 0.96(0.91-1.00) \\
\text { postmenopause } 0.94(0.91-0.97) \\
\text { overall: } 0.97(0.96-0.99) \\
\end{array}$ & $/ /$ \\
\hline$\underset{[47]}{\text { Mulholland }} 2008$ & $\begin{array}{c}\text { pre- and } \\
\text { post-menopause }\end{array}$ & $\begin{array}{l}\text { glycemic index (GI) and } \\
\text { glycemic load (GL) }\end{array}$ & $\begin{array}{l}\text { no strong associations between high } \\
\text { vs. low GI and BC risk; a positive } \\
\text { association emerged once analysis was } \\
\text { restricted to more robust measures of } \\
\text { dietary intake, for both premenopausal } \\
\text { and postmenopausal women. }\end{array}$ & // & $\begin{array}{c}\text { summary RR in cohort studies } \\
\text { (high vs. low GI) } \\
\text { premenopause RR } 1.20 \\
(1.01-1.43) \\
\text { postmenopause RR } 1.10 \\
(1.02-1.19)\end{array}$ & // \\
\hline
\end{tabular}


Table 2. Cont.

\begin{tabular}{|c|c|c|c|c|c|c|}
\hline Author_Year & Menopausal Status & Exposure Measure & Overall Results of Review & Statistical Method & $\begin{array}{c}\text { Summary Estimates and } \\
\text { Related } 95 \% \text { CI }\end{array}$ & $p$-value \\
\hline Mullie 2016 [48] & $\begin{array}{c}\text { pre- and } \\
\text { post-menopause }\end{array}$ & $\begin{array}{c}\text { dietary pattern with high GI } \\
\text { or GL }\end{array}$ & $\begin{array}{l}\text { women with high GI or GL had a } \\
\text { small, } 5-6 \% \text { increase in BC risk. }\end{array}$ & random effects model & $\begin{array}{c}\text { summary RR (high vs. low) for } \\
\text { GI } 1.05(1.00-1.11) \\
\text { - premenopause } 1.04(0.86-1.27) \\
\text { - postmenopause } 1.05(0.98-1.13) \\
\text { for GL 1.06 }(1.00-1.13) \\
\text { - premenopause } 1.23(0.75-2.00) \\
\text { - postmenopause } 1.05(0.97-1.13)\end{array}$ & // \\
\hline $\begin{array}{l}\text { Schwingshackl } \\
2014[15]\end{array}$ & // & $\begin{array}{l}\text { Mediterranean diet (MD; } \\
\text { fruits, vegetables, legumes, } \\
\text { olive oil, nuts, plant protein } \\
\text { and whole grains, fish; } \\
\text { moderate red wine } \\
\text { consumption and low } \\
\text { amounts of red meat, poultry } \\
\text { and dairy products) }\end{array}$ & $\begin{array}{l}\text { case-control studies showed a } \\
\text { significant association between greater } \\
\text { adherence to MD and lower BC risk; } \\
\text { cohort studies showed no significant } \\
\text { association between BC risk and MD. } \\
\text { Overall, MD was not associated with a } \\
\text { significant reduction in BC risk. }\end{array}$ & random effects model & $\begin{array}{l}\text { pooled RR (highest vs. lowest } \\
\text { adherence to MD category): } \\
\text { total } 0.95(0.84-1.06) \\
\text { cohort } 1.01(0.88-1.16) \\
\text { case-control } 0.82(0.69-0.97)\end{array}$ & $\begin{array}{l}0.35 \\
0.89 \\
0.02 \\
\end{array}$ \\
\hline Seely 2005 [42] & // & $\begin{array}{l}\text { green tea (dried leaves of } \\
\text { Camellia sinensis with } \\
\text { minimal oxidation of herbs, } \\
\text { polyphenols and catechins) }\end{array}$ & $\begin{array}{c}\text { random effects model revealed no } \\
\text { significant association between green } \\
\text { tea consumption and } B C \text { risk; fixed } \\
\text { effects model in case-control studies } \\
\text { showed a significant inverse } \\
\text { association. }\end{array}$ & $\begin{array}{l}\text { random effects model } \\
\text { fixed effects model }\end{array}$ & $\begin{array}{l}\text { pooled RR (highest vs. lowest } \\
\text { consumption) } \\
\text { cohort studies } 0.89(0.71-1.10) \\
\text { case-control studies } 0.44 \\
(0.14-1.31) \\
\text { case-control studies } 0.57 \\
\quad(0.38-0.86)\end{array}$ & $\begin{array}{l}0.28 \\
0.14 \\
0.007\end{array}$ \\
\hline Si 2014 [25] & $\begin{array}{c}\text { pre- and } \\
\text { post-menopause }\end{array}$ & $\begin{array}{l}\text { egg consumption quartiles: } \\
\begin{array}{c}1 \text { a week (reference) } \\
\geq 1,<2 \text { a week } \\
\geq 2, \leq 5 \text { a week } \\
>5 \text { a week }\end{array}\end{array}$ & $\begin{array}{l}\text { higher egg consumption was } \\
\text { associated with higher BC risk; } \\
\text { subgroup analyses showed this } \\
\text { association in cohort studies, among } \\
\text { Europeans, Asian populations, } \\
\text { postmenopausal women, and } \\
\text { consumers of } \geq 2 \text { and } \leq 5 \text { eggs/week. }\end{array}$ & random effects model & $\begin{array}{c}\text { pooled adjusted RR } \\
\text { overall 1.04 }(1.01-1.08) \\
\text { case control 1.06 }(0.97-1.15) \\
\text { cohort 1.04 }(1.00-1.08) \\
\text { postmenopause } 1.06(1.02-1.10) \\
\text { premenopause } 1.04(0.98-1.11) \\
\geq 2 \text { and } \leq 5 / \text { week }(\text { ref. }<1 / \text { week }) \\
\text { RR 1.10 }(1.02-1.17)\end{array}$ & $\begin{array}{c}0.02 \\
0.21 \\
0.05 \\
0.01 \\
0.2 \\
0.01\end{array}$ \\
\hline Song 2013 [40] & not stated & $\begin{array}{l}\text { citrus fruit intake, yellow and } \\
\text { orange fruit, oranges or } \\
\text { tangerines }\end{array}$ & $\begin{array}{l}\text { a higher intake of citrus fruit may } \\
\text { reduce BC risk. }\end{array}$ & fixed effects model & $\begin{array}{l}\text { summary OR (highest vs. lowest } \\
\text { intake group): } 0.90 \text { (0.85-0.96) }\end{array}$ & // \\
\hline
\end{tabular}


Table 2. Cont

\begin{tabular}{|c|c|c|c|c|c|c|}
\hline Author_Year & Menopausal Status & Exposure Measure & Overall Results of Review & Statistical Method & $\begin{array}{l}\text { Summary Estimates and } \\
\text { Related } 95 \% \text { CI }\end{array}$ & $p$-value \\
\hline Taylor 2009 [20] & premenopause & red meat consumption & $\begin{array}{l}\text { a significant positive association was } \\
\text { found overall between high of red meat } \\
\text { intake and BC risk among } \\
\text { premenopausal women; case-control } \\
\text { studies confirmed a strong association } \\
\text { between red meat and BC risk, while } \\
\text { cohort studies found no statistically } \\
\text { significant association between them. }\end{array}$ & // & $\begin{array}{l}\text { summary RR (highest vs. lowest } \\
\text { red meat intake): } \\
\text { overall } 1.24(1.08-1.42) \\
\text { cohort studies } 1.11(0.94-1.31) \\
\text { case-control studies } 1.57 \\
\quad(1.23-1.99)\end{array}$ & // \\
\hline Trock 2006 [31] & $\begin{array}{l}\text { pre- and } \\
\text { post-menopause }\end{array}$ & $\begin{array}{l}\text { soy protein (grams of soy } \\
\text { protein consumed daily) }\end{array}$ & $\begin{array}{l}\text { among all women, high soy intake was } \\
\text { modestly associated with a lower BC } \\
\text { risk; the association was not significant } \\
\text { for Asian women; among } 10 \text { studies } \\
\text { stratified by menopausal status, the } \\
\text { inverse association between soy } \\
\text { exposure and BC risk was stronger } \\
\text { premenopause than postmenopause. }\end{array}$ & $\begin{array}{l}\text { random effects model } \\
\text { and fixed effects } \\
\text { model (in } \\
\text { premenopausal } \\
\text { women) }\end{array}$ & $\begin{array}{l}\text { pooled OR (high vs. low soy } \\
\text { intake) } \\
\text { all women } 0.86(0.75-0.99) \\
\text { premenopause } 0.70(0.58-0.85) \\
\text { postmenopause } 0.77(0.60-0.98)\end{array}$ & // \\
\hline Turner 2011 [45] & $\begin{array}{l}\text { premenopause (7), } \\
\text { postmenopause (20), } \\
\text { and both (25) }\end{array}$ & $\begin{array}{c}\text { total fat, saturated fat (SAT), } \\
\text { monounsaturated fat } \\
\text { (MUFA), polyunsaturated fat } \\
\text { (PUFA) }\end{array}$ & $\begin{array}{l}\text { cohort studies found significant } \\
\text { summary RR between PUFA and BC; } \\
\text { case-control studies found no } \\
\text { association between fat and BC; } \\
\text { postmenopausal women had a } \\
\text { significant association between total fat, } \\
\text { PUFA intake and BC; an inverse } \\
\text { association between total intake of all } \\
\text { fats and BC was seen in premenopausal } \\
\text { women, but was not significant. }\end{array}$ & random effects model & $\begin{array}{l}\text { pooled RR (highest vs. lowest } \\
\text { quartile of consumption) } \\
\text { total fat } 1.01(0.99-1.03) \\
\text { SAT } 1.00(0.95-1.05) \\
\text { MUFA } 0.99(0.95-1.05) \\
\text { PUFA } 1.06(1.01-1.14) \\
\text { premenopause } 0.97(0.94-1.01) \\
\text { postmenopause } 1.04(1.01-1.07)\end{array}$ & $\begin{array}{l}\text { non- } \\
\text { significant } \\
\text { (NS) } \\
\text { NS } \\
\text { NS } \\
0.03 \\
\text { NS } \\
0.004\end{array}$ \\
\hline \multirow[t]{2}{*}{$\begin{array}{c}\text { Velentzis } 2009 \\
{[32]}\end{array}$} & \multirow[t]{2}{*}{$\begin{array}{l}\text { pre- and } \\
\text { post-menopause }\end{array}$} & \multirow[t]{2}{*}{ plant lignan intake } & $\begin{array}{l}\text { no significant association between plant } \\
\text { lignan intake and BC risk, despite a } \\
\text { slight protective effect; when } \\
\text { menopausal status was considered, a } \\
\text { statistically significant risk reduction }\end{array}$ & \multirow[t]{2}{*}{ random effects model } & $\begin{array}{l}\text { combined OR (highest vs. lowest } \\
\text { plant lignan intake) }\end{array}$ & \\
\hline & & & $\begin{array}{l}\text { was seen for the highest plant lignan } \\
\text { intake in postmenopausal women. }\end{array}$ & & $\begin{array}{c}\text { overall } 0.93(0.83-1.03) \\
\text { postmenopause } 0.85(0.78-0.93)\end{array}$ & $\begin{array}{c}0.15 \\
<0.001\end{array}$ \\
\hline
\end{tabular}


Table 2. Cont

\begin{tabular}{|c|c|c|c|c|c|c|}
\hline Author_Year & Menopausal Status & Exposure Measure & Overall Results of Review & Statistical Method & $\begin{array}{l}\text { Summary Estimates and } \\
\text { Related } 95 \% \text { CI }\end{array}$ & $p$-value \\
\hline Wu 2008 [9] & $\begin{array}{c}\text { pre- and } \\
\text { post-menopause }\end{array}$ & $\begin{array}{c}\text { soy intake - isoflavone } \\
\text { Asian (highest }>20 \mathrm{mg} / \text { day - } \\
\text { lowest }<5 \mathrm{mg} / \text { day) } \\
\text { Western (highest }>0.8 \mathrm{mg} / \text { day } \\
\text { - lowest }<0.15 \mathrm{mg} / \text { day) }\end{array}$ & $\begin{array}{l}\text { a statistically significant } 29 \% \text { reduction } \\
\text { in BC risk associated with high soy } \\
\text { intake in Asian populations (high } \\
\text { consumers); both premenopausal and } \\
\text { postmenopausal Asian women } \\
\text { consuming large amounts of soy had a } \\
\text { lower BC risk; no significant association } \\
\text { was found in Western countries. }\end{array}$ & random effects model & $\begin{array}{c}\text { pooled OR (highest vs. lowest } \\
\text { soy intake) } \\
\text { Asian } 0.71(0.60-0.85) \\
\text { - premenopause } 0.65(0.50-0.85) \\
\text { - postmenopause } 0.63(0.46-0.85) \\
\text { Western } 1.04(0.97-1.11)\end{array}$ & // \\
\hline Wu 2015 [33] & // & $\begin{array}{l}\text { dietary factors: vegetables, } \\
\text { fruits, soy food, or dietary fat }\end{array}$ & $\begin{array}{l}\text { high consumption of fruits, vegetables } \\
\text { and soy foods was significantly } \\
\text { inversely associated with a lower BC risk } \\
\text { in Chinese women; higher fatty food } \\
\text { consumption appeared to be related to } \\
\text { higher risk of BC in Chinese women. }\end{array}$ & random effects model & $\begin{array}{c}\text { combined OR (high vs. low } \\
\text { intake) } \\
\text { vegetables } 0.77(0.62-0.96) \\
\text { fruit } 0.68(0.49-0.93) \\
\text { soy food } 0.68(0.50-0.93) \\
\text { fat } 1.15(1.01-1.30) \\
\end{array}$ & $\begin{array}{l}0.02 \\
0.02 \\
0.02 \\
0.03\end{array}$ \\
\hline Yang 2014 [49] & $\begin{array}{c}\text { pre- and } \\
\text { post-menopause }\end{array}$ & $\begin{array}{l}\text { dietary polyunsaturated fatty } \\
\text { acid intake (n-3/n-6 PUFA) }\end{array}$ & $\begin{array}{l}\text { intake ratio of n-3/n- } 6 \text { PUFA was } \\
\text { inversely associated with BC risk for the } \\
\text { highest vs. lowest quantiles of the study } \\
\text { population. }\end{array}$ & random effects model & $\begin{array}{c}\text { pooled RR (highest quantile vs. } \\
\text { lowest) } \\
\text { overall diet } 0.90(0.82-0.99) \\
\text { premenopause } 0.80(0.43-1.48) \\
\text { postmenopause } 0.85(0.75-0.97) \\
\end{array}$ & // \\
\hline Zang 2015 [27] & $\begin{array}{c}\text { pre- and } \\
\text { post-menopause }\end{array}$ & $\begin{array}{c}\text { dairy food consumption: } \\
\text { high }(>600 \mathrm{~g} / \text { day }) \\
\text { modest }(400-600 \mathrm{~g} / \text { day }) \\
\text { low }(<400 \mathrm{~g} / \text { day) } \\
\text { dairy food items (milk, } \\
\text { cheese, butter) converted into } \\
\text { grams of protein per day }\end{array}$ & $\begin{array}{l}\text { higher dairy food consumption was } \\
\text { associated with a statistically significant } \\
\text { lower risk of } \mathrm{BC} \text {, in both cohort and } \\
\text { case-control studies; there was a } \\
\text { marginally lower BC risk in } \\
\text { premenopausal women. }\end{array}$ & random effects model & $\begin{array}{c}\text { combined estimates (highest } \\
\text { dairy consumption vs. lowest) } \\
\text { cohort RR } 0.90(0.83-0.98) \\
\text { - premenopause } 0.88(0.77-1.00) \\
\text { - postmenopause } 0.94(0.86-1.02) \\
\text { case-control OR } 0.74(0.62-0.88)\end{array}$ & // \\
\hline Zheng 2013 [50] & $\begin{array}{c}\text { pre- and } \\
\text { post-menopause }\end{array}$ & $\begin{array}{l}\text { dietary intake of marine } \mathrm{n}-3 \\
\text { polyunsaturated fatty acids } \\
\text { (PUFA) }\end{array}$ & $\begin{array}{l}\text { greater marine n-3 PUFA intake was } \\
\text { associated with a } 14 \% \text { reduction in BC } \\
\text { risk; no significant association for fish } \\
\text { intake or exposure to alpha-linoleic acid. }\end{array}$ & random effects model & $\begin{array}{c}\text { fish intake (highest vs. lowest } \\
\text { category) } \\
\text { overall 1.03 }(0.93-1.14) \\
\text { - premenopause } 1.04(0.91-1.20) \\
\text { - postmenopause 1.08 }(0.92-1.27) \\
\text { marine n-3 PUFA (highest vs. } \\
\text { lowest) } \\
\text { overall 0.86 }(0.78-0.94) \\
\text { - premenopause } 0.96(0.78-1.18) \\
\text { - postmenopause } 0.88(0.76-1.00)\end{array}$ & // \\
\hline
\end{tabular}


Table 2. Cont

\begin{tabular}{|c|c|c|c|c|c|c|}
\hline Author_Year & Menopausal Status & Exposure Measure & Overall Results of Review & Statistical Method & $\begin{array}{c}\text { Summary Estimates and } \\
\text { Related } 95 \% \text { CI }\end{array}$ & $p$-value \\
\hline Zhou 2015 [51] & $\begin{array}{c}\text { pre- and } \\
\text { post-menopause }\end{array}$ & linoleic acid & $\begin{array}{l}\text { both dietary linoleic acid and overall } \\
\text { linoleic acid level were associated with a } \\
\text { lower risk of BC, but none of the } \\
\text { associations were statistically significant. }\end{array}$ & $\begin{array}{l}\text { random and fixed } \\
\text { effects model }\end{array}$ & $\begin{array}{c}\text { exposure assessment (highest vs. } \\
\text { lowest category of linoleic acid) } \\
\text { overall } 0.98(0.93-1.04) \\
\text { dietary linoleic acid } 0.96 \\
(0.92-1.01) \\
\text { premenopause } 0.64(0.20-2.09) \\
\text { postmenopause } 1.01(0.94-1.08)\end{array}$ & // \\
\hline \multicolumn{7}{|c|}{ Results of pooled analyses included in the umbrella review (4) } \\
\hline $\begin{array}{c}\text { Mannisto } 2005 \\
{[16]}\end{array}$ & // & $\begin{array}{l}2 \text { dietary patterns with a high } \\
\text { intake of: VEG = vegetables, } \\
\text { legumes, fruit, pasta fish and } \\
\text { oil; PPP = pork, processed } \\
\text { meat, potatoes, beef, rice, } \\
\text { poultry, liver, butter/low-fat } \\
\text { margarine, pasta and coffee }\end{array}$ & $\begin{array}{l}\text { no significant association between VEG } \\
\text { pattern and BC risk in any of the cohorts } \\
\text { included in the analysis; in the } \\
\text { multivariate model PPP was not } \\
\text { significantly associated with BC risk in } \\
\text { two cohorts (ORDET, SMC), while in the } \\
\text { NLCS (Netherlands) cohort there was a } \\
\text { significantly inverse association between } \\
\text { PPP intake and BC risk (RR } \\
\text { 0.90_0.81-0.99). }\end{array}$ & // & // & // \\
\hline \multirow[t]{2}{*}{$\underset{[22]}{\text { Missmer } 2002}$} & \multirow[t]{2}{*}{$\begin{array}{c}\text { pre- and } \\
\text { post-menopause }\end{array}$} & \multirow[t]{2}{*}{$\begin{array}{l}\text { total meat, red and white } \\
\text { meat, dairy fluids and solids }\end{array}$} & \multirow{2}{*}{$\begin{array}{l}\text { no significant association between total } \\
\text { meat, red meat, white meat, total dairy } \\
\text { fluids or solids and BC risk. }\end{array}$} & \multirow[t]{2}{*}{ random effects model } & $\begin{array}{c}\text { pooled RR (highest vs. lowest } \\
\text { quartile) } \\
\text { total meat } 1.08(0.98-1.19) \\
\text { red meat } 0.94(0.87-1.02)\end{array}$ & $\begin{array}{l}0.13 \\
0.13\end{array}$ \\
\hline & & & & & $\begin{array}{l}\text { white meat } 1.02(0.91-1.13) \\
\text { dairy fluids } 0.93(0.84-1.03) \\
\text { dairy solids } 1.01(0.93-1.09)\end{array}$ & $\begin{array}{l}0.21 \\
0.09 \\
0.94\end{array}$ \\
\hline
\end{tabular}


Table 2. Cont.

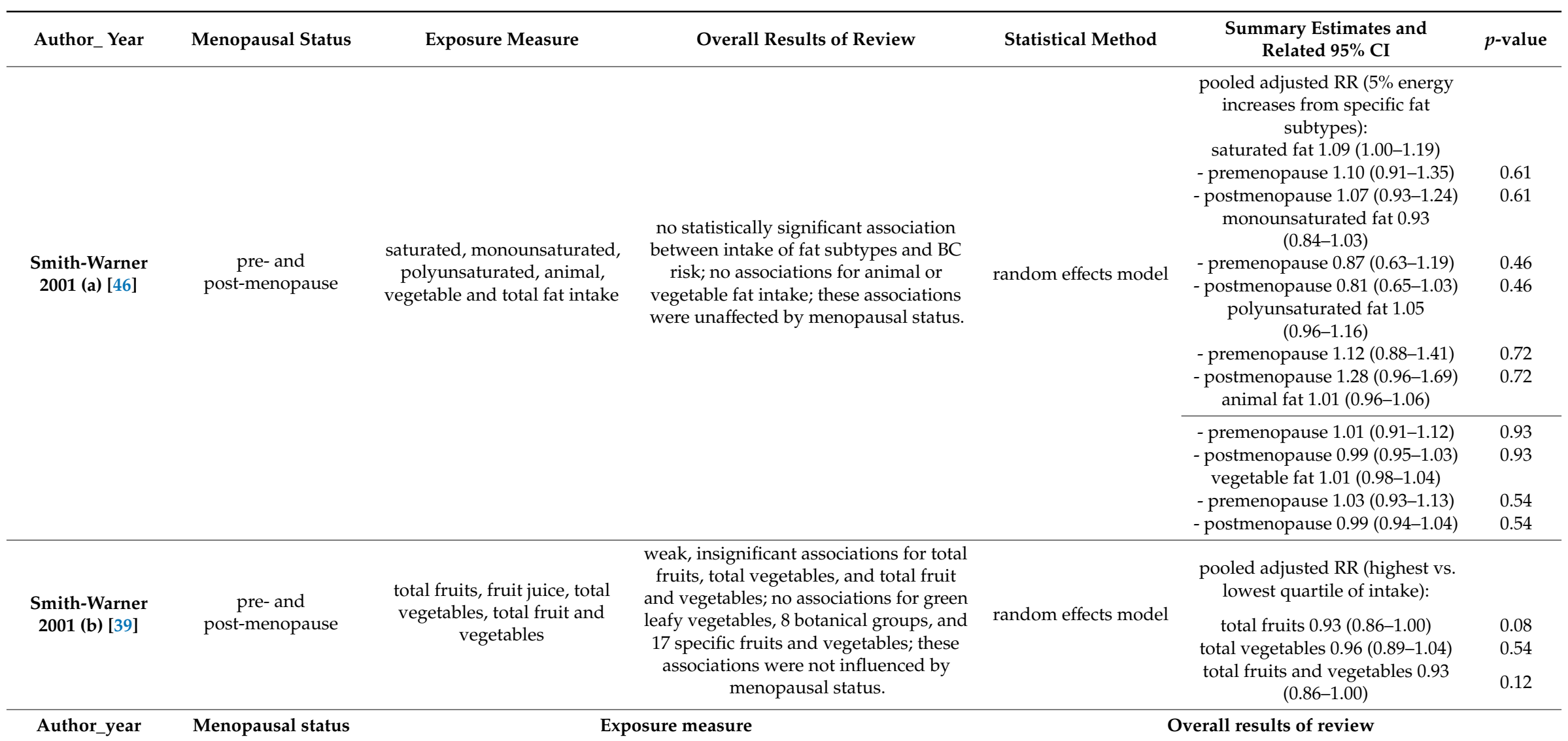


Table 2. Cont

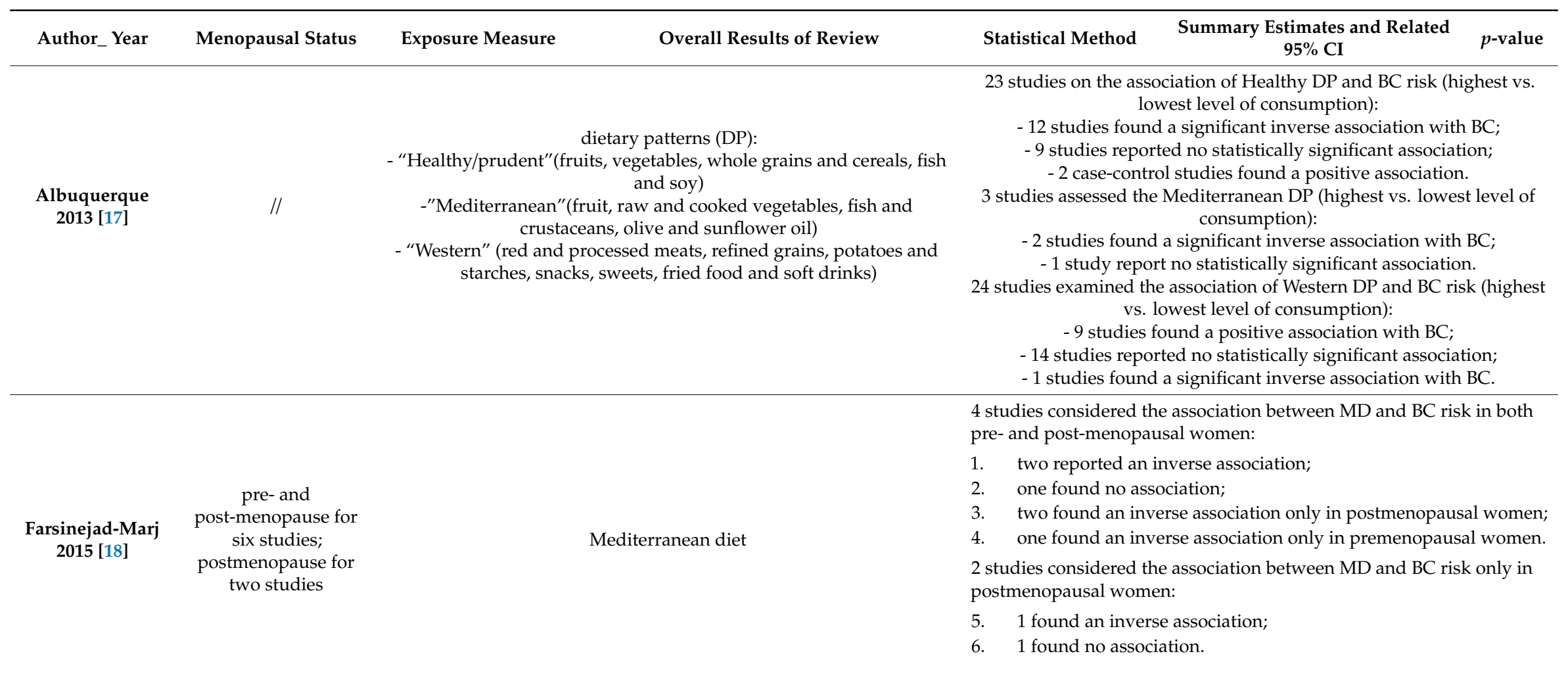

overall fat intake was unrelated to the incidence of BC; 2 cohorts

dietary exposures: fat intake, fruit and vegetables consumption, antioxidants, vitamins (A, C, E, $\beta$-carotene), carbohydrates, glycemic index (GI) and glycemic load (GL), dairy food consumption

Michels 2007 [28] pre- and post-menopause (including vitamin D), soy and isoflavones, green tea, heterocyclic amines, adolescent diet showed a significant positive association between high fat intake and BC risk (ORDET cohort: RR 3.47_1.43-8.44);

fruit and vegetable consumption may prevent $\mathrm{BC}$ : only one significant association (Nurses' Health Study) reported an inverse association for 5 or more vegetables/day;

vitamins A, C, E beta-carotene: no consistent association (except for vitamin $\mathrm{E}$ intake in postmenopausal women);

carbohydrates, GI and GL: no significant association

results for vitamin $D$, dairy and soy products were inconsistent. 
Table 2. Cont

\begin{tabular}{|c|c|c|c|}
\hline Author_Year & Menopausal Status & Overall Results of Review & $\begin{array}{l}\text { Summary Estimates and } \\
\text { Related } 95 \% \text { CI }\end{array}$ \\
\hline $\begin{array}{l}\text { Mourouti } 2013 \\
{[34]}\end{array}$ & // & soy consumption (isoflavones and lignans) & $\begin{array}{l}13 \text { out of } 16 \text { case-control studies, } 3 \text { out of } 3 \text { nested case-control studies, } \\
\text { and } 3 \text { out of } 10 \text { cohort studies demonstrated an inverse association } \\
\text { between soy food, soy product or isoflavone intake and BC risk; some } \\
\text { studies, } 3 \text { out of } 16 \text { case-control studies and } 6 \text { out of } 10 \text { cohort studies } \\
\text { found no association between soy or isoflavone intake and BC risk; } \\
\text { only } 1 \text { of } 29 \text { studies revealed a possible association between } \\
\text { phytoestrogen dietary intake and BC risk. }\end{array}$ \\
\hline $\begin{array}{l}\text { Mourouti } 2014 \\
{[21]}\end{array}$ & $\begin{array}{c}\text { pre- and } \\
\text { post-menopause }\end{array}$ & $\begin{array}{l}\text { intake of fruit and vegetables, meat, soy products and isoflavones, dietary } \\
\text { fiber, dietary carbohydrates, dietary lipids }\end{array}$ & $\begin{array}{l}\text { for fruit and vegetable consumption, most studies found a significant } \\
\text { inverse association with BC risk; } \\
\text { for meat intake, } 7 \text { studies found a significantly higher risk and } 7 \text { found } \\
\text { no association; most case-control studies on soy consumption found } \\
\text { an inverse association; } \\
\text { most studies on dietary fiber found no association; } \\
3 \text { cohort and } 2 \text { case-control studies on dietary carbohydrates found a } \\
\text { higher BC risk for higher GI and GL, while the other } 11 \text { studies found } \\
\text { no association; } \\
6 \text { out of } 10 \text { case-control studies found a positive association with high } \\
\text { fat intake, but most cohort studies found no significant association. }\end{array}$ \\
\hline \multicolumn{4}{|c|}{ Results of qualitative reviews included in the umbrella review (7) } \\
\hline Cui 2006 [54] & $\begin{array}{c}\text { pre- and } \\
\text { post-menopause }\end{array}$ & $\begin{array}{l}\text { dietary and supplemental vitamin D intake; dietary and supplemental } \\
\text { calcium intake }\end{array}$ & $\begin{array}{l}7 \text { studies ( } 5 \text { on pre- and post-menopausal women, } 1 \text { on } \\
\text { postmenopausal women, } 1 \text { on premenopausal women) of the } 12 \\
\text { studies considered found a statistically significant inverse association } \\
\text { (highest vs. lowest category of intake) between calcium intake and BC } \\
\text { risk; this study showed no association between dietary and } \\
\text { supplemental vitamin D intake and BC; only one cohort study found a } \\
\text { protective effect among premenopausal women. }\end{array}$ \\
\hline Duffy 2007 [35] & $\begin{array}{c}\text { pre- and } \\
\text { post-menopause }\end{array}$ & phytoestrogen intake & $\begin{array}{l}10 \text { case-control studies showed a significant inverse association } \\
\text { between phytoestrogen/isoflavone/lignin intake and BC risk, while } 5 \\
\text { revealed no such association; only } 1 \text { of } 6 \text { cohort studies found a } \\
\text { protective effect of high isoflavone intake. }\end{array}$ \\
\hline $\begin{array}{c}\text { Eichholzer } 2001 \\
\text { [57] }\end{array}$ & $\begin{array}{c}\text { pre- and } \\
\text { post-menopause }\end{array}$ & dietary folate intake & $\begin{array}{l}\text { epidemiological studies suggested an inverse association between } \\
\text { folate intake and BC among female regular alcohol consumers; no } \\
\text { significant association between folate intake and BC risk in the overall } \\
\text { population. }\end{array}$ \\
\hline
\end{tabular}


Table 2. Cont.

\begin{tabular}{|c|c|c|c|}
\hline Author_Year & Menopausal Status & Overall Results of Review & $\begin{array}{l}\text { Summary Estimates and } \\
\text { Related } 95 \% \text { CI }\end{array}$ \\
\hline Hanf 2005 [23] & // & $\begin{array}{l}\text { fat intake; meat and fish consumption; fruit and vegetable consumption, } \\
\text { fiber consumption }\end{array}$ & $\begin{array}{l}12 \text { studies tested total fat intake as a risk factor for BC, and only one } \\
\text { showed a statistically significant reduced RR with a higher total fat } \\
\text { intake; animal fat was not found associated with BC; no significant } \\
\text { association emerged for saturated, monounsaturated and } \\
\text { polyunsaturated fatty acids: } 4 / 10 \text { prospective studies found a positive } \\
\text { correlation between high levels of meat consumption and BC risk; no } \\
\text { association between fish intake and BC; no significant correlation } \\
\text { between BC and high consumption of fruit and vegetables; no overall } \\
\text { association between fiber intake and BC risk: only } 1 \text { of } 5 \text { studies } \\
\text { suggested an effect of fiber. }\end{array}$ \\
\hline Lof 2006 [36] & $\begin{array}{l}\text { pre- and } \\
\text { post-menopause }\end{array}$ & dietary lignan intake & $\begin{array}{l}4 \text { of } 5 \text { case-control studies showed a protective effect of dietary lignans } \\
\text { against BC, especially in premenopausal women; } 2 \text { prospective } \\
\text { studies did not confirm any protective effect of dietary lignans. }\end{array}$ \\
\hline Peeters 2003 [37] & $\begin{array}{l}\text { pre- and } \\
\text { post-menopause }\end{array}$ & $\begin{array}{c}\text { phytoestrogens (soybeans, soy products, miso, tofu, soy protein, } \\
\text { isoflavones) }\end{array}$ & $\begin{array}{l}\text { overall, results showed no protective effect of dietary intake of soy } \\
\text { products against BC, except for women who consumed } \\
\text { phytoestrogens in adolescence or in very high doses. }\end{array}$ \\
\hline Rossi 2014 [60] & // & $\begin{array}{l}\text { proteins, carbohydrates and glycemic index (GI), dietary fat, polyphenols } \\
\text { and phytoestrogens, fruits and vegetables, lycopene, vitamins and oligo } \\
\text { elements, alcohol }\end{array}$ & $\begin{array}{l}\text { consumption of well- or over-cooked red meat was associated with a } \\
\text { higher risk of BC; } \mathrm{n}-3 \text { PUFA may protect against BC; a lower GI seems } \\
\text { associated with a lower risk of BC; high-fat diet, and high total } \\
\text { cholesterol and triglyceride levels are associated with a higher risk of } \\
\text { BC; evidence supports a protective role of lycopene, polyphenols, fruit } \\
\text { and vegetables against BC; there was evidence of vitamin D reducing } \\
\text { the BC risk; and of zinc and, to a lesser extent, vitamins E and B } \\
\text { reducing the BC risk thanks to their antioxidant properties. }\end{array}$ \\
\hline
\end{tabular}


Table 3. Conclusions and limitations of studies included in the umbrella review.

\begin{tabular}{|c|c|c|c|c|}
\hline Author_Year & Limitations & Recommendations/Conclusions & Quality Assessment Tool & Quality Assessment \\
\hline \multicolumn{5}{|c|}{ Conclusion and quality assessment of meta-analysis included in the umbrella review (32) } \\
\hline Alexander 2010 [43] & $\begin{array}{l}\text { Meta-analysis limited to analysis of results reported } \\
\text { across publications. Many studies on fat intake and } \\
\text { BC probably did not examine fat from animal } \\
\text { sources alone. Differential reporting of dietary } \\
\text { factors may influence summary associations across } \\
\text { the literature. The association between dietary fat } \\
\text { and BC subgroups is also incomplete. }\end{array}$ & $\begin{array}{l}\text { The available epidemiological evidence from } \\
\text { prospective studies does not support an } \\
\text { independent association between animal fat intake } \\
\text { and } \mathrm{BC} \text { risk. Better dietary assessments and } \\
\text { methods in cohort studies, also examining dietary } \\
\text { fat and BC in randomized clinical trials, may help to } \\
\text { clarify any possible relationships. }\end{array}$ & $/ /$ & // \\
\hline Aune 2012 [38] & $\begin{array}{l}\text { Potential unmeasured or residual confounders; a } \\
\text { higher fiber intake is often associated with other } \\
\text { lifestyle factors (higher levels of physical activity, } \\
\text { lower prevalence of obesity, lower intake of alcohol } \\
\text { and dietary fat); measurement errors in dietary } \\
\text { intake assessments are known to bias } \\
\text { effect estimates. }\end{array}$ & $\begin{array}{l}\text { Diet with a high intake of plant-based foods rich in } \\
\text { fiber could have an impact in the prevention of } B C \text {; } \\
\text { this review suggests that diets rich in fiber are } \\
\text { associated with a lower BC risk. }\end{array}$ & // & // \\
\hline Boyd 2003 [44] & $\begin{array}{l}\text { Measurement error in the food frequency } \\
\text { questionnaires (FFQs) used in most studies may lead } \\
\text { to overestimation of the range of intakes, and may } \\
\text { also lead to attenuation of risk. }\end{array}$ & $\begin{array}{l}\text { Combined risk estimates of for total and saturated } \\
\text { fat intake, and for meat intake, all indicated an } \\
\text { association between higher intakes and an increased } \\
\text { risk of BC. Case-control and cohort studies gave } \\
\text { similar results. }\end{array}$ & $\begin{array}{l}\text { Quality of studies scored } \\
\text { using preset } \\
\text { methodological standards }\end{array}$ & $\begin{array}{l}\text { Twenty-six studies } \\
\text { defined as } \\
\text { "higher-quality" }\end{array}$ \\
\hline Brennan 2010 [14] & $\begin{array}{l}\text { Possible recall bias in case-control combined } \\
\text { analysis; possible weakness in design of the studies } \\
\text { (different response rates and inconsistent adjustment } \\
\text { for potential confounders). Possible misclassification } \\
\text { within the two dietary patterns. }\end{array}$ & $\begin{array}{l}\text { This review provided evidence of a small inverse } \\
\text { association between a healthy diet and a positive } \\
\text { trend between Western diet and BC risk. The results } \\
\text { of this meta-analysis highlight the need for more } \\
\text { carefully designed observational and intervention } \\
\text { studies to clarify the influence of diet on BC risk. }\end{array}$ & // & // \\
\hline Buck 2010 [29] & $\begin{array}{l}\text { Potential measurement errors due to recall bias or } \\
\text { shortcomings in food composition database. }\end{array}$ & $\begin{array}{l}\text { High lignan exposure may be associated with a } \\
\text { reduced risk of BC in postmenopausal women. } \\
\text { Additional work is warranted to confirm } \\
\text { this association. }\end{array}$ & $/ /$ & // \\
\hline Chen 2010 [52] & Not reported. & $\begin{array}{l}\text { From the meta-analysis, there was a significant } \\
\text { inverse association between vitamin D and calcium } \\
\text { intake and BC risk; these findings support the use of } \\
\text { vitamin D and calcium as chemopreventive agents } \\
\text { against BC. Better designed clinical trials are needed } \\
\text { to ascertain the protective effect and optimize the } \\
\text { doses of these nutrients. }\end{array}$ & $/ /$ & // \\
\hline
\end{tabular}


Table 3. Cont

\begin{tabular}{|c|c|c|c|c|}
\hline Author_Year & Limitations & Recommendations/Conclusions & Quality Assessment Tool & Quality Assessment \\
\hline Chen 2014 [55] & $\begin{array}{l}\text { The BC prevention effect of folate may be conferred by } \\
\text { other nutrients in foods (leafy vegetables, legumes, } \\
\text { egg yolk, baker's yeast); dose-effect relationships for } \\
\text { dietary folate intake level and BC risk differed } \\
\text { between prospective and case-control studies; } \\
\text { changing dietary habits during the follow-up in } \\
\text { prospective studies was not assessed; differences in } \\
\text { sample size, study region, study design and } \\
\text { assessment methods may cause heterogeneity and } \\
\text { prompt differences in the stratification analysis. }\end{array}$ & $\begin{array}{l}\text { The findings revealed a potential non-linear dose } \\
\text { effect for dietary folate levels against BC risk, and } \\
\text { modest folate intake may reduce BC risk. Given the } \\
\text { complexity of folate metabolism and uses, the dose } \\
\text { and timing of folate intake should be considered. } \\
\text { More prospective studies are necessary. }\end{array}$ & $\begin{array}{l}\text { Newcastle-Ottawa Scale } \\
\text { (NOS) }\end{array}$ & $\begin{array}{l}\text { Thirty studies } \\
\text { classified as } \\
\text { "higher-quality" } \\
(\geq 5 \text { points (pt)), twelve } \\
\text { as "lower-quality" } \\
(<5 \mathrm{pt})\end{array}$ \\
\hline Dong 2011 (a) [30] & $\begin{array}{c}\text { Strong degree of heterogeneity made results difficult } \\
\text { to interpret. Changes in soy consumption during the } \\
\text { long follow-up may have weakened the } \\
\text { associations observed. }\end{array}$ & $\begin{array}{l}\text { A statistically significant inverse association was } \\
\text { confirmed between soy isoflavone intake and } \mathrm{BC} \\
\text { risk. The protective effect of soy against } \mathrm{BC} \text { may be } \\
\text { due to other, associated healthy lifestyle factors (high } \\
\text { vegetable and fruit intake, more physical activity). }\end{array}$ & // & $/ /$ \\
\hline Dong 2011 (b) [26] & $\begin{array}{l}\text { Consumption of dairy food, especially low-fat } \\
\text { products, is probably associated with a healthy } \\
\text { lifestyle; the likelihood of inadequate control for } \\
\text { confounding factors may bias the findings; dietary } \\
\text { assessments suffer from measurement error; possible } \\
\text { misclassification bias; substantial heterogeneity } \\
\text { across studies. }\end{array}$ & $\begin{array}{l}\text { Findings indicate that a higher consumption of total } \\
\text { dairy products may be associated with a reduced } \mathrm{BC} \\
\text { risk: menopausal status may influence this effect. }\end{array}$ & $/ /$ & $/ /$ \\
\hline Gandini 2000 [24] & $\begin{array}{c}\text { Possibility of publication bias (due to studies not being } \\
\text { published if findings are not significant); } \\
\text { heterogeneous intake categories. }\end{array}$ & $\begin{array}{l}\text { This analysis confirmed the association between } \\
\text { vegetable and, to a lesser extent, fruit intake and BC } \\
\text { risk. Increasing vegetable consumption, and the } \\
\text { intake of associated micronutrients (vitamin C and } \\
\text { b-carotene) might reduce the risk of BC. }\end{array}$ & $/ /$ & $/ /$ \\
\hline Gissel 2008 [53] & $\begin{array}{l}\text { Few studies and low vitamin } \mathrm{D} \text { intake reported in } \\
\text { most trials; possible bias from diet reporting. }\end{array}$ & $\begin{array}{c}\text { Increasing the intake of vitamin D above } 400 \mathrm{IU} / \text { day } \\
\text { may help to prevent BC; but more research is } \\
\text { needed. }\end{array}$ & $/ /$ & $/ /$ \\
\hline Guo 2015 [19] & $\begin{array}{l}\text { The residual confounders in the studies are a problem } \\
\text { in the meta-analysis of observational studies; these } \\
\text { findings are probably affected by the misclassification } \\
\text { of meat; the intake and consumption levels in the } \\
\text { highest and lowest categories varied across studies; } \\
\text { possibility of publication bias. }\end{array}$ & $\begin{array}{l}\text { A high intake of red and/or processed meat was } \\
\text { associated with an increased risk of BC. Additional } \\
\text { cohort or interventional studies will be needed to } \\
\text { confirm the association. }\end{array}$ & $/ /$ & $/ /$ \\
\hline
\end{tabular}


Table 3. Cont

\begin{tabular}{|c|c|c|c|c|}
\hline Author_Year & Limitations & Recommendations/Conclusions & Quality Assessment Tool & Quality Assessment \\
\hline Hu 2012 [59] & $\begin{array}{l}\text { Socio-economic position may influence the intake of } \\
\text { carotenoids; the lack of original data in the studies } \\
\text { limited the assessment of potential interactions; no } \\
\text { cohort study in Asia focused on the associations } \\
\text { between carotenoids and BC. This analysis did not } \\
\text { assess the quality of all the studies included. }\end{array}$ & $\begin{array}{l}\text { Total } \alpha \text {-carotene intake could reduce the } \mathrm{BC} \text { risk. } \\
\text { The relationship between } \beta \text {-carotene intake and } \mathrm{BC} \\
\text { needs to be confirmed. No significant associations } \\
\text { emerged between dietary } \beta \text {-cryptoxanthin, } \\
\text { lutein+zeaxanthin, lycopene and BC. }\end{array}$ & $/ /$ & $/ /$ \\
\hline Hui 2013 [58] & // & $\begin{array}{l}\text { The study suggested that the intake of flavonols and } \\
\text { flavones, but not of other flavonoid subclasses, is } \\
\text { associated with a decreased risk of BC, especially } \\
\text { after menopause. }\end{array}$ & // & // \\
\hline Larsson $2007[56]$ & $\begin{array}{l}\text { Potential bias due to confounders inherent in the } \\
\text { original studies; potential recall, interviewer or } \\
\text { selection bias in case-control studies; heterogeneity } \\
\text { due to methodological differences among the studies. }\end{array}$ & $\begin{array}{l}\text { No clear support for an overall relationship between } \\
\text { folate intake and BC risk. Adequate folate intake } \\
\text { may have a protective effect against BC. }\end{array}$ & // & // \\
\hline Li 2014 [41] & $\begin{array}{l}\text { Relatively small number of eligible studies included in } \\
\text { the analysis, and only two prospective studies; limited } \\
\text { information extracted from the original studies; } \\
\text { different types of mushroom. }\end{array}$ & $\begin{array}{l}\text { The findings suggested that a higher edible } \\
\text { mushroom consumption may be associated with a } \\
\text { lower risk of BC; large-scale prospective studies are } \\
\text { needed to confirm as much. }\end{array}$ & $\begin{array}{l}\text { Newcastle-Ottawa Scale } \\
\text { (NOS)—9 pt } \\
\text { scoring system }\end{array}$ & $\begin{array}{l}\text { Four studies classified } \\
\text { as "higher-quality" } \\
\quad(\geq 7 \mathrm{pt}) \\
\text { three as } \\
\text { "lower-quality" (<7 pt) }\end{array}$ \\
\hline $\begin{array}{l}\text { Mulholland } 2008 \\
{[47]}\end{array}$ & $\begin{array}{l}\text { Study results inconsistently adjusted for potential } \\
\text { confounders might result in residual confounding } \\
\text { elements; a relatively small number of studies } \\
\text { included in the analysis, making it difficult to estimate } \\
\text { publication bias and heterogeneity. }\end{array}$ & $\begin{array}{l}\text { High dietary GI and GL do not appear to be of } \\
\text { etiological importance in BC development. }\end{array}$ & $\begin{array}{l}\text { Newcastle-Ottawa quality } \\
\text { assessment Scale (NOS) } \\
\text { — pt scoring system }\end{array}$ & $\begin{array}{l}\text { Fourteen } \\
\text { "higher-quality" } \\
\text { studies }\end{array}$ \\
\hline Mullie 2016 [48] & $\begin{array}{l}\text { Not all studies reported women's menopausal status. } \\
\text { Lack of RR unadjusted and adjusted for adiposity. }\end{array}$ & $\begin{array}{l}\text { The evidence supports a modest association between } \\
\text { a dietary pattern with high GI/GL and the risk of BC. }\end{array}$ & // & // \\
\hline $\begin{array}{l}\text { Schwingshackl } 2014 \\
\text { [15] }\end{array}$ & $\begin{array}{l}\text { Relationship between diet and cancer is complex } \\
\text { (multiple confounding variables); Mediterranean diet } \\
\text { (MD) was not homogenous (heterogeneity on score } \\
\text { items); possible measurement errors and } \\
\text { recall/selection bias in the studies included. }\end{array}$ & $\begin{array}{l}\text { No significant changes in risk reduction for } \mathrm{BC} \text { by } \\
\text { adherence to } \mathrm{MD} \text {. }\end{array}$ & $\begin{array}{l}\text { Newcastle-Ottawa quality } \\
\text { assessment Scale } \\
\text { (NOS)—9 pt scoring } \\
\text { system }\end{array}$ & $\begin{array}{l}\text { Eight "higher-quality" } \\
(\geq 7 \mathrm{pt}) \text { and three } \\
\text { "lower-quality" } \\
\text { studies }(<7 \mathrm{pt})\end{array}$ \\
\hline Seely 2005 [42] & $\begin{array}{l}\text { Small number of observational studies and no RCTs; } \\
\text { marked variability among questionnaire response } \\
\text { rates in cohort studies; unadjusted analysis for } \\
\text { potential confounding factors; possible selection bias } \\
\text { that could distort the findings; case-control studies } \\
\text { produced weaker evidence than cohort studies. }\end{array}$ & $\begin{array}{l}\text { This systematic review and meta-analysis found no } \\
\text { significant effect of green tea on } B C \text { prevention. } \\
\text { Green tea consumption may be associated with a } \\
\text { reduced risk of BC recurrences. }\end{array}$ & // & // \\
\hline
\end{tabular}


Table 3. Cont.

\begin{tabular}{|c|c|c|c|c|}
\hline Author_Year & Limitations & Recommendations/Conclusions & Quality Assessment Tool & Quality Assessment \\
\hline Si 2014 [25] & $\begin{array}{l}\text { Only articles written in English were included in the } \\
\text { meta-analysis; possible selection bias; categorical } \\
\text { standards for egg consumption were confused, making } \\
\text { it difficult to them among the studies; cooking methods } \\
\text { were not ascertained. }\end{array}$ & $\begin{array}{l}\text { Pooled results of cohort studies and combined } \\
\text { results showed that higher egg consumption was } \\
\text { associated with a higher BC risk, especially for } \\
\text { European and postmenopausal populations. More } \\
\text { studies should be conducted using a well-recognize } \\
\text { categorical standard for egg consumption. Egg } \\
\text { consumption should be reduced to reduce BC risk. }\end{array}$ & // & // \\
\hline Song 2013 [40] & $\begin{array}{l}\text { Diversity of study design; the apparent association of } \\
\text { these pooled results is restricted to the case-control } \\
\text { study (overestimating the exposure effect due to recall } \\
\text { and selection bias); measurement error in the FFQs; } \\
\text { cut-off points for citrus fruit intake vary across studies, } \\
\text { so there is uncertainty about the optimal citrus fruit } \\
\text { intake for the prevention of BC. }\end{array}$ & $\begin{array}{c}\text { Pooled results from observational studies showed an } \\
\text { inverse association between citrus fruit intake and } \\
\text { the risk of BC. This study underscores the need for } \\
\text { well-designed prospective observational and } \\
\text { intervention studies to clarify the influence of citrus } \\
\text { fruit on BC. }\end{array}$ & // & // \\
\hline Taylor 2009 [20] & $\begin{array}{l}\text { Bias involving the use of FFQs in all studies (self-report } \\
\text { measures to assess consumption can lead to } \\
\text { misclassification of intake); recall bias in case-control } \\
\text { studies may affect observed associations between } \\
\text { dietary intake and cancer risk (tendency to over-report } \\
\text { among cases and under-report among controls). }\end{array}$ & $\begin{array}{l}\text { Red meat may be a source of heterocyclic amines } \\
\text { and heme-iron, a highly bioavailable form of iron } \\
\text { which has been shown to enhance estrogen-induced } \\
\text { tumor formation. This quantitative summary of } \\
\text { published literature indicates that red meat may } \\
\text { contribute to BC risk in premenopausal women. }\end{array}$ & // & // \\
\hline Trock 2006 [31] & $\begin{array}{l}\text { Possible measurement errors among the studies; } \\
\text { potential selection bias; different levels of soy } \\
\text { consumption among the studies; potentially } \\
\text { uncontrolled confounders among the studies. }\end{array}$ & $\begin{array}{l}\text { Soy intake may be associated with a small reduction } \\
\text { in BC risk. This result should be interpreted with } \\
\text { caution, however, due to potential exposure } \\
\text { misclassification, confounders, or lack of a } \\
\text { dose-response effect. }\end{array}$ & // & // \\
\hline Turner 2011 [45] & $\begin{array}{c}\text { Small sample size in premenopausal studies; association } \\
\text { between BC risk and PUFA subtypes not } \\
\text { examined separately. }\end{array}$ & $\begin{array}{c}\text { High-fat diet may increase estrogen levels and } \\
\text { therefore increase BC risk. Higher risk of BC in } \\
\text { postmenopausal women consuming large amounts } \\
\text { of total fat and PUFA. Conversely, dietary fat may } \\
\text { have some BC prevention effects in } \\
\text { premenopausal women. }\end{array}$ & $/ /$ & // \\
\hline Velentzis 2009 [32] & $\begin{array}{l}\text { Over- or under-estimation of food content; some food } \\
\text { composition databases are incomplete; the amount of } \\
\text { lignans in food can differ depending on crop variety, } \\
\text { location, year of harvest and processing; possible dietary } \\
\text { measurement errors associated with FFQs; possibility of } \\
\text { residual confounding factors. }\end{array}$ & $\begin{array}{l}\text { Plant lignans may be associated with a small }(15 \%) \\
\text { reduction in postmenopausal BC risk, but further } \\
\text { studies are needed to confirm these results. }\end{array}$ & // & $/ /$ \\
\hline
\end{tabular}


Table 3. Cont

\begin{tabular}{|c|c|c|c|c|}
\hline Author_Year & Limitations & Recommendations/Conclusions & Quality Assessment Tool & Quality Assessment \\
\hline Wu 2008 [9] & Not reported & $\begin{array}{l}\text { Overall, data based on Asian women showed a } \\
\text { dose-dependent statistically significant association } \\
\text { between soy food intake and BC risk reduction; soy } \\
\text { intake was unrelated to BC risk in studies conducted } \\
\text { on Western populations. }\end{array}$ & $/ /$ & $/ /$ \\
\hline Wu 2015 [33] & $\begin{array}{l}\text { Different units used to assess food intakes among the } \\
\text { studies; dose-response analysis was not conducted; } \\
\text { possible measurement errors and analysis } \\
\text { unadjusted for potential confounding factors. }\end{array}$ & $\begin{array}{l}\text { High fruit and vegetable consumption, as a } \\
\text { protective measure, can reduce the incidence of BC } \\
\text { in Chinese women. High fruit consumption protects } \\
\text { against BC (higher soy food consumption induces a } \\
69 \% \text { reduction in BC risk). High-fat food } \\
\text { consumption may increase BC risk. }\end{array}$ & $\begin{array}{l}\text { Newcastle-Ottawa quality } \\
\text { assessment Scale } \\
\text { (NOS)—9 pt scoring } \\
\text { system }\end{array}$ & $\begin{array}{l}\text { Eleven studies were } \\
\text { "higher-quality" } \\
(\geq 7 \mathrm{pt}) \text { and eleven } \\
\text { were "lower-quality" } \\
\quad(<7 \mathrm{pt})\end{array}$ \\
\hline Yang 2014 [49] & $\begin{array}{l}\text { Potential publication bias (small studies with null } \\
\text { results tend not to be published); any measurement } \\
\text { error and resulting misclassification would most } \\
\text { likely lead to an attenuation of the true association; } \\
\text { dietary questionnaires are liable to measurement } \\
\text { errors (reporting bias or inaccurate databases); } \\
\text { potential residual confounding factors not excluded. }\end{array}$ & $\begin{array}{l}\text { Findings provide conclusive evidence to support } \\
\text { increasing n-3/n-6 PUFA intake for BC prevention. } \\
\text { For every } 1 / 10 \text { increment in n-3/n-6 PUFA there was } \\
\text { a } 6 \% \text { reduction in BC risk; importance of promoting } \\
\text { nutritional education (increasing the consumption of } \\
\text { marine foods). }\end{array}$ & $\begin{array}{l}\text { Newcastle-Ottawa quality } \\
\text { assessment Scale } \\
\text { (NOS)—9 pt scoring } \\
\text { system }\end{array}$ & $\begin{array}{c}\text { Six studies were } \\
\text { classified as } \\
\text { "high-quality" }(\geq 8 \mathrm{pt}), \\
\text { five as } \\
\text { "moderate-quality" } \\
(<8 \mathrm{pt})\end{array}$ \\
\hline Zang 2015 [27] & $\begin{array}{l}\text { Potential confounding factors inherent in the } \\
\text { original study design; misclassification of dairy food } \\
\text { consumption (self-reporting system) may cause } \\
\text { incorrect estimates; potential methodological } \\
\text { differences between studies; use of case-control } \\
\text { studies may provide a lower level of evidence. }\end{array}$ & $\begin{array}{l}\text { Dairy food consumption was inversely associated } \\
\text { with BC risk; possible association between high } \\
\text { dairy food consumption and beneficial } \\
\text { environmental factors (economic status, physical } \\
\text { activity, healthier lifestyle). }\end{array}$ & $\begin{array}{l}\text { Newcastle-Ottawa quality } \\
\text { assessment Scale } \\
\text { (NOS)—9 pt scoring } \\
\text { system }\end{array}$ & $\begin{array}{l}\text { Twelve studies scored } \\
8 \text { pts, nine scored } 7 \mathrm{pts}, \\
\text { and six scored } 6 \text { pts }\end{array}$ \\
\hline Zheng 2013 [50] & $\begin{array}{l}\text { Different assessment methods; limited data available } \\
\text { on individual n-3 PUFA; potential residual } \\
\text { confounders not analyzed; possible language bias. }\end{array}$ & $\begin{array}{c}\text { This meta-analysis provided strong and solid } \\
\text { evidence of marine n-3 PUFA intake being inversely } \\
\text { associated with risk of BC. }\end{array}$ & $\begin{array}{l}\text { Newcastle-Ottawa quality } \\
\text { assessment Scale } \\
\text { (NOS)—9 pt scoring } \\
\text { system }\end{array}$ & $\begin{array}{l}\text { Studies were of } \\
\text { "moderate-quality" } \\
(5-7 \mathrm{pt}) \text { and } \\
\text { "higher-quality" } \\
(>7 \mathrm{pt})\end{array}$ \\
\hline Zhou 2015 [51] & $\begin{array}{l}\text { Selection bias might be unavoidable; relatively } \\
\text { limited amount of data included in the } \\
\text { meta-analysis; potential measurement errors and } \\
\text { misclassification; unmeasured or residual } \\
\text { confounders might affect the association between } \\
\text { linoleic acid and BC risk }\end{array}$ & $\begin{array}{l}\text { This meta-analysis suggested that linoleic acid is } \\
\text { associated with a lower risk of } \mathrm{BC} \text {, but none of the } \\
\text { associations were statistically significant. }\end{array}$ & $\begin{array}{l}\text { Newcastle-Ottawa quality } \\
\text { assessment Scale } \\
\text { (NOS)—9 pt scoring } \\
\text { system }\end{array}$ & $\begin{array}{l}\text { Four studies scored } \\
6 / 9 \text { pts, ten studies } \\
\text { scored } 7 / 9 \text { pts }\end{array}$ \\
\hline
\end{tabular}


Table 3. Cont

\begin{tabular}{|c|c|c|c|c|}
\hline Author_Year & Limitations & Recommendations/Conclusions & Quality Assessment Tool & Quality Assessment \\
\hline \multicolumn{5}{|c|}{ Conclusion and quality assessment of pooled analysis included in the umbrella review (4) } \\
\hline Mannisto 2005 [16] & // & $\begin{array}{l}\text { The results supported the suggestion deriving from } \\
\text { relatively recent epidemiological research that diet } \\
\text { may not have an important role in the etiology of BC. } \\
\text { The possible protective effect of "Pork, Processed } \\
\text { Meat, Potatoes" dietary pattern in the NLCS cohort } \\
\text { could be explained by a difference in that pattern for } \\
\text { NLCS (type of food included in the FFQs). }\end{array}$ & // & // \\
\hline Missmer 2002 [22] & $\begin{array}{l}\text { Inability to assess the effect of how food was cooked } \\
\text { and for how long (data not collected); possible } \\
\text { measurement errors not collected. }\end{array}$ & $\begin{array}{l}\text { This large study does not provide evidence of a diet } \\
\text { rich in meat and dairy products increasing the risk } \\
\text { of BC. }\end{array}$ & // & // \\
\hline $\begin{array}{l}\text { Smith-Warner } 2001 \\
\text { (a) [46] }\end{array}$ & $\begin{array}{l}\text { Possible errors in estimates of consumption (over- or } \\
\text { under-estimation); possible methodological errors in } \\
\text { study design. }\end{array}$ & $\begin{array}{l}\text { The pooled analysis suggested only a weak positive } \\
\text { association for replacing saturated fat consumption } \\
\text { with carbohydrate consumption; none of the other } \\
\text { types of fat examined were associated with BC risk; } \\
\text { these results, though not significant, are compatible } \\
\text { with a lower BC risk observed for higher intakes of } \\
\text { olive oil (rich source of monounsaturated fat) in } \\
\text { studies conducted in Mediterranean countries. }\end{array}$ & // & // \\
\hline $\begin{array}{l}\text { Smith-Warner } 2001 \\
\text { (b) [39] }\end{array}$ & $\begin{array}{l}\text { Differences in study design; numerous fruit and } \\
\text { vegetable categories reported in the studies; possible } \\
\text { publication bias. }\end{array}$ & $\begin{array}{l}\text { The results suggest that fruit and vegetable } \\
\text { consumption in adulthood is not significantly } \\
\text { associated with BC risk (BC risk only } 3 \% \text { to } 9 \% \text { lower } \\
\text { in women in the highest decile of fruit or vegetable } \\
\text { consumption). No specific fruits or vegetables had } \\
\text { strong and significant association with BC risk. }\end{array}$ & $/ /$ & // \\
\hline \multicolumn{5}{|c|}{ Conclusion and quality assessment of systematic reviews included in the umbrella review (5) } \\
\hline $\begin{array}{c}\text { Albuquerque } 2013 \\
{[17]}\end{array}$ & $\begin{array}{l}\text { No summary estimate measure was produced; } \\
\text { instruments used to collect dietary information } \\
\text { differed among the studies; FFQs, used in most cases, } \\
\text { are liable to measurement error and might not detect } \\
\text { a significant association; great variability among the } \\
\text { studies; possibility of recall or selection bias. }\end{array}$ & $\begin{array}{l}\text { The results suggest that a dietary pattern } \\
\text { characterized by vegetables, fruit, fish and soy } \\
\text { (healthy), or the Mediterranean diet reduces the risk } \\
\text { of BC. A positive association was observed for the } \\
\text { Western dietary pattern, but most of these results } \\
\text { were not statistically significant. Efforts are expected } \\
\text { to aid future research. }\end{array}$ & Strobe Checklist & // \\
\hline
\end{tabular}


Table 3. Cont.

\begin{tabular}{|c|c|c|c|c|}
\hline Author_Year & Limitations & Recommendations/Conclusions & Quality Assessment Tool & Quality Assessment \\
\hline $\begin{array}{l}\text { Farsinejad-Marj } \\
\quad 2015[18]\end{array}$ & $\begin{array}{l}\text { Scarcity of studies, no assessment of factors } \\
\text { hypothesized to relate to BC (breastfeeding, } \\
\text { adiposity, physical activity, genetic background, } \\
\text { insulin resistance, and chronic inflammation). Few } \\
\text { studies separately investigated risk of BC in pre- and } \\
\text { post-menopausal women. Different components of } \\
\text { the MD can act synergistically in reducing BC risk so } \\
\text { it is important to consider all components of the MD } \\
\text { in studies. }\end{array}$ & $\begin{array}{l}\text { Case-cohort studies found an inverse association } \\
\text { between the MD and BC risk in pre- and } \\
\text { post-menopausal women, but cohort studies } \\
\text { reported controversial results. It seems that there are } \\
\text { insufficient data to reach a conclusion about the } \\
\text { effect of MD on BC risk before and after menopause, } \\
\text { but there is some evidence to suggest a protective } \\
\text { association. More cohort studies need to be } \\
\text { conducted in different parts of the world to confirm } \\
\text { these results. }\end{array}$ & // & $/ /$ \\
\hline Michels 2007 [28] & $\begin{array}{l}\text { Possible measurement error (FFQs); great } \\
\text { heterogeneity in dietary patterns among different } \\
\text { countries; timing in diet; possible inadequate } \\
\text { follow-up in studies. }\end{array}$ & $\begin{array}{l}\text { There was no consistent strong and statistically } \\
\text { significant association between diet and BC risk. }\end{array}$ & $/ /$ & // \\
\hline Mourouti 2013 [34] & $\begin{array}{l}\text { Possible recall bias; variation in exposure measures } \\
\text { used in the studies; variation in the amount and type } \\
\text { of soy consumed and in the sample size may have } \\
\text { over- or under- estimated the true effect sizes } \\
\text { measured. Potential dietary measurement error may } \\
\text { mask the true relationship. }\end{array}$ & $\begin{array}{l}\text { The majority of the studies ( } 66 \% \text { ) revealed a } \\
\text { statistically significant inverse association between } \\
\text { soy and isoflavones consumption and BC risk. } \\
\text { Limitations in the study design may mask the real } \\
\text { associations. }\end{array}$ & $/ /$ & $/ /$ \\
\hline Mourouti 2014 [21] & $\begin{array}{l}\text { Differences in experimental design and habits } \\
\text { among study populations; lack of sufficient } \\
\text { follow-up in the studies; methodological problems } \\
\text { with the assessment tools used in each study. }\end{array}$ & $\begin{array}{l}\text { No consistent and statistically strong association } \\
\text { emerged between BC incidence and dietary factors; } \\
\text { soy food and isoflavone intake seemed to protect } \\
\text { against BC, mainly in Asian populations; no } \\
\text { association reported for the consumption of dietary } \\
\text { carbohydrates, GI or GL, dietary fiber intake and the } \\
\text { risk of BC. The consumption of dietary fat probably } \\
\text { raises the risk of BC. The studies that examined the } \\
\text { role of fruit, vegetable and meat intake provide } \\
\text { inconsistent results. }\end{array}$ & $/ /$ & $/ /$ \\
\hline
\end{tabular}


Table 3. Cont

\begin{tabular}{|c|c|c|c|c|}
\hline Author_Year & Limitations & Recommendations/Conclusions & Quality Assessment Tool & Quality Assessment \\
\hline \multicolumn{5}{|c|}{ Conclusion and quality assessment of qualitative reviews included in the umbrella review (7) } \\
\hline Cui $2006[54]$ & $\begin{array}{l}\text { Possible selection bias due to case-control study } \\
\text { design; shortcomings in methodology and summary } \\
\text { estimate measures. }\end{array}$ & $\begin{array}{l}\text { Despite inconsistent results from the } \\
\text { epidemiological studies, some evidence indicates } \\
\text { that vitamin D and calcium might have a protective } \\
\text { role against BC. Experimental evidence suggests that } \\
\text { the association between vitamin D intake and BC } \\
\text { may be stronger for premenopausal than for } \\
\text { postmenopausal women. Further analyses are } \\
\text { required to assess this interaction. }\end{array}$ & $/ /$ & // \\
\hline Duffy 2007 [35] & Not reported & $\begin{array}{l}\text { Early exposure in childhood or adolescence may } \\
\text { have a protective effect against BC. }\end{array}$ & // & // \\
\hline Eichholzer 2001 [57] & Not reported & $\begin{array}{l}\text { High folate intake may be associated with a lower } \\
\text { risk of BC in women at high risk (alcohol users). }\end{array}$ & // & // \\
\hline Hanf 2005 [23] & Not reported & $\begin{array}{l}\text { Regional differences in } \mathrm{BC} \text { incidence are probably } \\
\text { partially attributable to lifelong dietary habits. }\end{array}$ & // & // \\
\hline Lof $2006[36]$ & $\begin{array}{l}\text { Potential misclassification of phytoestrogen intake } \\
\text { due to measurement associated with dietary } \\
\text { assessment; potential recall bias in retrospective } \\
\text { studies. }\end{array}$ & $\begin{array}{l}\text { Epidemiological data seem to support a small } \\
\text { protective effect of isoflavones against BC; as for } \\
\text { lignans and BC, the results are somewhat } \\
\text { inconsistent, but the protective effect might be } \\
\text { limited to premenopausal women. }\end{array}$ & // & // \\
\hline Peeters 2003 [37] & $\begin{array}{l}\text { None of the studies considered age at time of } \\
\text { consumption, which seems to be important in BC } \\
\text { development; methodological differences between } \\
\text { the studies are too large to estimate any summary } \\
\text { effect; limited number of epidemiological studies on } \\
\text { phytoestrogens and BC. }\end{array}$ & $\begin{array}{l}\text { None of the studies found evidence for an increased } \\
\text { risk of } B C \text { with an increased phytoestrogen intake. }\end{array}$ & // & // \\
\hline Rossi 2014 [60] & $\begin{array}{l}\text { Limitations due to study design (methodological } \\
\text { shortcomings and lack of association measures). }\end{array}$ & $\begin{array}{l}\text { Nutritional factors might have a role in the } \\
\text { development of BC; red meat intake and dietary } \\
\text { sugar should be reduced, whereas increased fish } \\
\text { intake appears to be protective; fruit and vegetables, } \\
\text { including polyphenols, have shown promising } \\
\text { results. Prospective randomized trials are needed to } \\
\text { develop population-based prevention strategies } \\
\text { for BC. }\end{array}$ & // & // \\
\hline
\end{tabular}




\section{Discussion}

Judging from this review, a higher intake of total meat, or red or processed meats, or of foods with a high GI, or eggs would seem to be associated with a higher risk of BC. Other foods, such as vegetables, soy and carotenoids, would seem to be inversely associated with BC risk. One meta-analysis demonstrated an inverse association between BC and the consumption of citrus fruits and mushrooms. Some nutrients also seem to be inversely associated with BC risk, including calcium, folate, vitamin D, lignans, and carotenoids. As concerns exposure to other dietary elements (polyunsaturated fatty acids, dairy foods), the evidence is still conflicting.

\subsection{Dietary Patterns}

Our findings indicate that most of the literature analyzed here attributed a protective role to the MD [19,51]. Research has shown that this type of diet is rich in antioxidants, which probably inhibit the synthesis and activity of growth factors promoting the development of cancer cells. A more recent meta-analysis of studies on postmenopausal BC [61] found an inverse association between MD and BC risk, when alcohol was excluded (summary Hazard Ratio (HR) 0.92; 95\% CI 0.87, 0.98), while this association disappeared when alcohol was included. This might indicate that the MD could even be used as a primary BC prevention measure, especially in postmenopausal women. Some authors have said that calorie balance, adiposity control, and exercise are important to BC prevention too, as well as the composition and quality of the diet [62-65]. The MD also seems to have a beneficial influence against the risk of BC regardless of body weight and BMI [66-69]. The literature on the traditional MD has demonstrated that dietary fiber has multiple protective effects, which include inhibiting intestinal estrogen reabsorption, and modulating cholesterol levels and glucose release, thereby reducing BC risk [70]. The protective effect of fruit and vegetables seems to be linked to their high content of beneficial substances (vitamins, minerals, salicylates, phytosterols, glucosinolates, polyphenols, phytoestrogens, sulfides, lectins, etc.), which have an antioxidant action, preventing the activation of many carcinogens, suppressing spontaneous mutations, and protecting cellular structures and DNA against the oxidative damage generated by metabolic processes [70-72]. Leafy vegetables are rich in lutein, zeaxanthin, folates, vitamin A and carotenoids, which are antioxidant and also able to regulate estrogen metabolism and inhibit tumor growth $[73,74]$. Fruits seem to have an anti-carcinogenic potential due to their antioxidant properties; this is especially true of red fruits, which contain ellagic acid, quercitin and anthocyanins. These substances stimulate the mechanisms behind the elimination of toxic substances, inhibit angiogenesis, reduce inflammation, and promote cellular apoptosis mechanisms [71,75]. Some mushroom-derived substances, like polysaccharides, are known for their anti-tumor and immunomodulatory properties, for enhancing immune system activity and protecting against tumor recurrences [76]. A pooled analysis of eight large cohort studies did not strongly associate the intake of fruit and vegetables with the risk of BC, however [46]. Another comparative study of three cohorts also indicated that a diet rich in vegetables and fruits, but also characterized by other foods such as oil and fish, was not significantly related to a lower risk of BC [46].

The studies included in the present review indicated that the Western diet, which involves a high intake of refined sugars, saturated fats and alcohol, is strongly associated with an increased risk of BC $[14,17]$. This type of diet influences inflammatory processes, and induces an increase in adiposity and the production of growth factors and hormones (estrogen and testosterone).

Overall, however, the studies reviewed did not produce consistent results concerning specific dietary patterns and the risk of BC. Further studies could probably be conducted using machine learning techniques: this type of analysis could test clusters of foods not defined a priori-instead of prototypic dietary patterns-in terms of their association with BC risk, or BC prevention. In fact, a diet can include numerous foods that might have opposite effects, in which case analyzing preset eating patterns would be unable to produce evidence of any clear association. 


\subsection{Foods}

The significant association demonstrated by the EPIC study [77] between red or processed meat consumption and a higher risk of BC, and BC-related mortality, was also confirmed in our umbrella review $[19,20]$. Apart from the related fat intake, various other factors appear to be involved in the carcinogenic potential of red and processed meat: the presence of carcinogens or their precursors (polycyclic aromatic hydrocarbons, heterocyclic aromatic amines, nitrosamines) produced by food treatments like cooking, salting or smoking; a high intake of animal proteins, with a potential increase in insulin-like growth factor (IGF) levels; hormone residues contained in meat from intensive farming; and the fact that intestinal fermentation of animal proteins raises the concentration of some polyamines, which are fundamental to cell proliferation.

A significant association also emerged between egg consumption and a higher risk of BC [24], but this relationship needs to be confirmed. The most plausible mechanism behind the carcinogenic potential of eggs might relate to their high cholesterol content (425 mg per $100 \mathrm{~g}$ ) [24], given the recommended daily dose of $300 \mathrm{mg}$. Cholesterol is a precursor of steroid hormones, so it can influence estrogen activity and contribute to cellular inflammation, a crucial component in BC progression [78]. However, some evidence points to the association between eggs and BC being not necessarily due to cholesterol [79]: when fried at high temperatures, eggs also become a source of heterocyclic amines and several carcinogenic components, so their mutagenic activity can increase in some conditions (cooking at high temperatures and with inappropriate oil) [80].

Our umbrella review showed that studies consistently provided evidence of a protective effect of soy in the diet against the risk of BC $[9,29,30,32,34-36]$, especially among postmenopausal women $[28,31,33]$. Phytoestrogens are structurally and functionally natural substances similar to estradiol, with a similar estrogenic activity. The evidence indicates a lower BC risk in consumers of soybean and phytoestrogen-containing plants, particularly in Asian populations (who are exposed to such foods from childhood), and in postmenopausal women. The protective effect of these foods is due to the agonist or antagonist actions against estrogens in breast tissue, reducing blood levels of estradiol [81,82] and consequently also the risk of estrogen receptor positive $(\mathrm{ER}+)$ /progesterone receptor positive $(\mathrm{PR}+) \mathrm{BC}$.

Finally, our umbrella review found that consuming lean dairy products was associated with a lower risk of BC $[25,26]$, probably due to the protective effect of vitamin D and calcium [52-54]. Breast tissue has receptors to the biologically active form of vitamin $\mathrm{D}$, calcitriol $1,25(\mathrm{OH})_{2} \mathrm{D}$, which appears to be able to directly and indirectly control more than 200 genes, including those responsible for cell proliferation, malignant cell differentiation, apoptosis and angiogenesis [83]. In contrast, there are also reports of dairy product consumption leading to an increased estrogen hormone intake, which can enhance the penetrance of BC associated with Breast Related Cancer Antigens (BRCA) mutations [84]. In addition, milk has the potential to raise blood levels of growth factors, and a diet rich in animal protein is associated with high serum IGF-1 levels, which are strongly associated with a greater risk of BC $[85,86]$.

The consumption of citrus fruits, rich in vitamin $C, \beta$-carotene, quercitin and folate, seems to have a very positive impact on health due to the antioxidant, immune-stimulant, and detoxifying properties, and a capacity to modulate insulin sensitivity and cholesterol levels [87]. In fact, our review found a $10 \%$ lower BC risk in women who consumed large amounts of citrus fruit.

\subsection{Nutrients}

A significant inverse association has been demonstrated between BC risk and consumption of carotenoids [88], which have antioxidant properties. These compounds, especially $\beta$-carotenes, are capable of binding and eliminating free radicals, and repairing DNA damage, inhibiting cell proliferation, inducing apoptosis, and suppressing angiogenesis [89]. Flavonoids have been acknowledged to have a role in protecting against and preventing non-communicable diseases [90-93], and neoplasms in particular, due to their potent antioxidant and DNA repairing activity. In this 
review, the dietary impact of various subclasses of flavonoids was analyzed, and it proved significant for flavonols, flavones, and flavan-3-ol swell classes, particularly after menopause. Folic acid, a soluble member of the vitamin B group, is needed for all DNA synthesis, repair and methylation reactions, so folate deficiency in the diet can negatively affect cell division and DNA repair mechanisms. The studies assessed in our umbrella review generally reported inconsistent results on the association between folic acid and BC, however.

Foods with a high GI, such as simple sugars, refined carbohydrates and starches, induce a rapid increase in blood glucose, and consequently stimulate insulin production. High levels of insulin cause the production of IGF-1 and testosterone, which are recognized as risk factors for BC. In addition, chronic hyperinsulinemia with associated insulin resistance has a key role in the etiology of $\mathrm{BC}$ as it induces the production of IGF-1, which is capable of causing mutagenic changes [94]. The studies included in our review $[47,48]$ demonstrated no strong association between high GI food consumption and any increased risk of $\mathrm{BC}$, however; this is probably due to reliability issues with the measurement indices adopted.

On the whole, our review identified a moderate risk of BC in women with a high total fat and/or saturated fat intake $[32,44,46]$. There was also evidence to support this higher BC risk applying especially to postmenopausal women [95-97], and the consumption of saturated fat is also associated with a higher likelihood of obesity [98-104]. Obesity and overweight, together with lack of exercise, are established risk factors for $\mathrm{BC}[105,106]$. A diet rich in saturated fat also increases estrogen synthesis, leading to an increased cell proliferation and a consequently higher BC risk [107,108].

On the other hand, our review found that polyunsaturated fatty acids (PUFA) can have a role in reducing BC risk [49,50], although some studies found controversial results [21,45]. A possible explanation of the discrepancy of results concerns the fact that the two studies that found a protective effect of the PUFAs against the BC specifically examined the intake of $n-3 / n-6$ PUFAs [49] and marine n-3 PUFA [50]. On the contrary, in the two studies [21,45] which gave an opposite result, the association between BC and the PUFA subtypes was not examined. The n-3 PUFA family compete for the same metabolic pathway with the n-6 PUFA family, which is associated with cell proliferation in breast tissue. The n-3 PUFA family reduces inflammation, controls triglyceride levels, and thus reduces the risk of BC via several mechanisms, i.e., by altering the composition of the phospholipid cell membranes, inhibiting arachidonic acid (ARA) metabolism and pro-inflammatory molecule production, and modulating the expression and function of various receptors, transcription factors, and signaling molecules [109]. Marine-derived n-3 PUFA would seem to have a protective effect against the development of BC [110], particularly in postmenopausal women, whereas n-6 PUFA contribute to carcinogenic mechanisms with the production of pro-inflammatory eicosanoids, such as prostaglandin E2, which is implicated in angiogenic processes and in the suppression of cancer cell apoptosis $[109,111]$.

Overall, our study confirms the content of the third report from the World Cancer Research Fund [112], which found moderate evidence of the following: consuming non-starchy vegetables might reduce the risk of estrogen-receptor-negative (ER-) BC; consuming foods containing carotenoids, or adopting diets high in calcium might reduce the risk of $\mathrm{BC}$ in both premenopausal and postmenopausal women; and consuming dairy products might reduce the risk of $\mathrm{BC}$, but only in premenopausal women. Our study also indicated that other foods and nutrients, including soy, folate, vitamin D, and lignans, seem to be inversely associated with BC.

This umbrella review of studies investigating the associations between diet, or specific foods or nutrients and BC reveals the weaknesses of the observational studies and reviews published on this topic (as shown in Table 3). The main shortcoming concerns measurement errors in dietary intake assessments, which bias the estimates of any such associations. Meta-analyses and systematic reviews of case-control studies may also be affected by recall and interview bias, often revealing associations that are not confirmed in cohort studies. Some studies also adjusted inconsistently for potential confounders (e.g., physical activity, often associated with both outcome and exposure), and this could result in residual confounding elements that would bias the estimates emerging from a meta-analysis. 


\section{Conclusions}

Nutrition is one of the most modifiable aspects of lifestyle, and nutritional choices can affect people's health and the risk of cancer. Active strategies are warranted, including educational/behavioral interventions in high-risk groups, to promote healthy eating habits.

Supplementary Materials: The following are available online at http://www.mdpi.com/1660-4601/17/13/4731/s1, Table S1: Summary of measures of BC risk associated with dietary exposures-For META-ANALYSES only; Table S2: Summary of measures of BC risk associated with dietary exposures; Table S3: Quality of studies included in the umbrella review.

Author Contributions: Study conception and design: A.B.; acquisition of data, review literature: M.P., L.L., G.G.; interpretation of data: G.G., A.B., V.B.; drafting of manuscript: G.G., L.L.; critical revision: A.B. All authors have read and agreed to the published version of the manuscript.

Funding: This research received no specific grant from any funding agency, commercial or not-for-profit sectors.

Conflicts of Interest: The authors have no financial or other relationships that might lead to a conflict of interest.

\section{References}

1. Tumore al Seno-Tumori AIRC. Available online: http://www.airc.it/tumori/tumore-al-seasp (accessed on 31 August 2017).

2. Schütze, M.; Boeing, H.; Pischon, T.; Rehm, J.; Kehoe, T.; Gmel, G.; Olsen, A.; Tjønneland, A.M.; Dahm, C.C.; Overvad, K.; et al. Alcohol attributable burden of incidence of cancer in eight European countries based on results from prospective cohort study. BMJ 2011, 342, 1-10. [CrossRef] [PubMed]

3. Sieri, S.; Chiodini, P.; Agnoli, C.; Pala, V.; Berrino, F.; Trichopoulou, A.; Benetou, V.; Vasilopoulou, E.; Sánchez, M.J.; Chirlaque, M.; et al. Dietary fat intake and development of specific breast cancer subtypes. J. Natl. Cancer Inst. 2014, 106, 5. [CrossRef] [PubMed]

4. Mattisson, E.; Wirfält, P.; Wallström, B.; Gullberg, H.O.L.; Berglund, G. High fat and alcohol intakes are risk factors of postmenopausal breast cancer: A prospective study from the Malmö diet and cancer cohort. Int. J. Cancer 2004, 110, 589-597. [CrossRef] [PubMed]

5. Zhang, C.-X.; Ho, S.C.; Lin, F.-Y.; Chen, Y.-M.; Cheng, S.-Z.; Fu, J.-H. Dietary fat intake and risk of breast cancer: A case-control study in China. Eur. J. Cancer Prev. 2011, 20, 199-206. [CrossRef]

6. Jansen, E.C.; Marin, C.; Mora-Plazas, M.; Villamor, E. Higher childhood red meat intake frequency is associated with earlier age at menarche. J. Nutr. 2016, 146, 792-798. [CrossRef]

7. Varinska, L.; Gal, P.; Mojzisova, G.; Mirossay, L.; Mojzis, J. Soy and breast cancer: Focus on angiogenesis. Int. J. Mol. Sci. 2015, 16, 11728-11749. [CrossRef]

8. Messina, M.; McCaskill-Stevens, W.; Lampe, J.W. Addressing the soy and breast cancer relationship: Review, commentary, and workshop proceedings. J. Natl. Cancer Inst. 2006, 98, 1275-1284. [CrossRef]

9. Wu, H.; Yu, M.C.; Tseng, C.C.; Pike, M.C. Epidemiology of soy exposures and breast cancer risk. Br. J. Cancer 2008, 98, 9-14. [CrossRef] [PubMed]

10. Aromataris, E.; Fernandez, R.; Godfrey, C.M.; Holly, C.; Khalil, H.; Tungpunkom, P. Summarizing systematic reviews. Int. J. Evid. Based Healthc. 2015, 13, 132-140. [CrossRef]

11. Aromataris, E.; Fernandez, R.; Godfrey, C.; Holly, C.; Khalil, H.; Tungpunkom, P. Methodology for JBI Umbrella Reviews. Joanna Briggs Inst. Rev. Man. 2014, 1-34. Available online: https://ro.uow.edu.au/cgi/ viewcontent.cgi?article $=4367 \&$ context=smhpapers (accessed on 29 June 2020).

12. Moher, D.; Liberati, A.; Tetzlaff, J.; Altman, D. PRISMA CHECKLIST. PLoS Med. 2009, 6, 7.

13. Moher, D.; Altman, D.G.; Liberati, A.; Tetzlaff, J. PRISMA Statement. Epidemiology 2011, 22, 1. [CrossRef] [PubMed]

14. Brennan, S.F.; Cantwell, M.M.; Cardwell, C.R.; Velentzis, L.S.; Woodside, J.V. Dietary patterns and breast cancer risk: A systematic review and meta-analysis. Am. J. Clin. Nutr. 2010, 91, 1294-1302. [CrossRef] [PubMed]

15. Schwingshackl, L.; Hoffmann, G. Adherence to Mediterranean diet and risk of cancer: A systematic review and meta-analysis of observational studies. Int. J. Cancer 2014, 135, 1884-1897. [CrossRef] 
16. Männistö, S.; Dixon, L.B.; Balder, H.F.; Virtanen, M.J.; Krogh, V.; Khani, B.R.; Berrino, F.; van den Brandt, P.A.; Hartman, A.M.; Pietinen, P.; et al. Dietary patterns and breast cancer risk: Results from three cohort studies in the DIETSCAN project. Cancer Causes Control 2005, 16, 725-733. [CrossRef] [PubMed]

17. Albuquerque, R.C.R.; Baltar, V.T.; Marchioni, D.M.L. Breast cancer and dietary patterns: A systematic review. Nutr. Rev. 2014, 72, 1-17. [CrossRef]

18. Farsinejad-Marj, M.; Talebi, S.; Ghiyasvand, R.; Miraghajani, M. Adherence to Mediterranean diet and risk of breast cancer in premenopausal and postmenopausal women. Arch. Iran. Med. 2015, 18, 786-792.

19. Guo, J.; Wei, W.; Zhan, L. Red and processed meat intake and risk of breast cancer: A meta-analysis of prospective studies. Breast Cancer Res. Treat. 2015, 151, 191-198. [CrossRef]

20. Taylor, V.H.; Misra, M.; Mukherjee, S.D. Is red meat intake a risk factor for breast cancer among premenopausal women? Breast Cancer Res. Treat. 2009, 117, 1-8. [CrossRef]

21. Mourouti, N.; Kontogianni, M.D.; Papavagelis, C.; Panagiotakos, D.B. Diet and breast cancer: A systematic review. Int. J. Food Sci. Nutr. 2015, 66, 1-42. [CrossRef]

22. Missmer, S.A.; Smith-Warner, S.A.; Spiegelman, D.; Yaun, S.S.; Adami, H.O.; Beeson, W.L.; Van Den Brandt, P.A.; Fraser, G.E.; Freudenheim, J.L.; Goldbohm, R.A.; et al. Meat and dairy food consumption and breast cancer: A pooled analysis of cohort studies. Int. J. Epidemiol. 2002, 31, 78-85. [CrossRef] [PubMed]

23. Hanf, V.; Gonder, U. Nutrition and primary prevention of breast cancer: Foods, nutrients and breast cancer risk. Eur. J. Obstet. Gynecol. Reprod. Biol. 2005, 123, 139-149. [CrossRef] [PubMed]

24. Gandini, S.; Merzenich, H.; Robertson, C.; Boyle, P. Meta-analysis of studies on breast cancer risk and diet: The role of fruit and vegetable consumption and the intake of associated micronutrients. Eur. J. Cancer 2000, 36, 636-646. [CrossRef]

25. Si, R.; Qu, K.; Jiang, Z.; Yang, X.; Gao, P. Egg consumption and breast cancer risk: A meta-analysis. Breast Cancer 2014, 21, 251-261. [CrossRef]

26. Dong, J.-Y.; Zhang, L.; He, K.; Qin, L.-Q. Dairy consumption and risk of breast cancer: A meta-analysis of prospective cohort studies. Breast Cancer Res. Treat. 2011, 127, 23-31. [CrossRef]

27. Zang, J.; Shen, M.; Du, S.; Chen, T.; Zou, S. The association between dairy intake and breast cancer in western and Asian populations: A systematic review and meta-analysis. J. Breast Cancer 2015, 18, 313-322. [CrossRef]

28. .Michels, K.B.; Mohllajee, P.A.; Roset-Bahmanyar, E.; Beehler, G.P.; Moysich, K.B. Diet and breast cancer: A review of the prospective observational studies. Cancer 2007, 109, 2712-2749. [CrossRef]

29. Buck, K.; Zaineddin, A.K.; Vrieling, A.; Linseisen, J.; Chang-Claude, J. Meta-analyses of lignans and enterolignans in relation to breast cancer risk. Am. J. Clin. Nutr. 2010, 92, 141-153. [CrossRef]

30. Dong, J.-Y.; Qin, L.-Q. Soy isoflavones consumption and risk of breast cancer incidence or recurrence: A meta-analysis of prospective studies. Breast Cancer Res. Treat. 2011, 125, 315-323. [CrossRef]

31. Trock, B.J.; Leena, H.C.; Clarke, R. Meta-analysis of soy intake and breast cancer risk. J. Natl. Cancer Inst. 2006, 98, 459-471. [CrossRef]

32. Velentzis, L.S.; Cantwell, M.M.; Cardwell, C.; Keshtgar, M.R.; Leathem, A.J.; Woodside, J.V. Lignans and breast cancer risk in pre- and post-menopausal women: Meta-analyses of observational studies. Br. J. Cancer 2009, 100, 1492-1498. [CrossRef]

33. Wu, Y.-C.; Zheng, D.; Sun, J.-J.; Zou, Z.-K.; Ma, Z.-L. Meta-analysis of studies on breast cancer risk and diet in Chinese women. Int. J. Clin. Exp. Med. 2015, 8, 73-85. [PubMed]

34. Mourouti, N.; Panagiotakos, D.B. Soy food consumption and breast cancer. Maturitas 2013, 76, 118-122. [CrossRef] [PubMed]

35. Duffy, C.; Perez, K.; Partridge, A. Implications of phytoestrogen intake for breast cancer. Cancer J. Clin. 2007, 57, 60-277. [CrossRef] [PubMed]

36. Lof, M.; Weiderpass, E. Epidemiologic evidence suggests that dietary phytoestrogen intake is associated with reduced risk of breast, endometrial, and prostate cancers. Nutr. Res. 2006, 26, 609-619. [CrossRef]

37. Peeters, P.H.M.; Keinan-Boker, L.; van der Schouw, Y.T.; Grobbee, D.E. Phytoestrogens and breast cancer risk. Breast Cancer Res. Treat. 2003, 77, 71-183. [CrossRef]

38. Aune, D.; Chan, D.S.; Greenwood, D.C.; Vieira, A.R.; Rosenblatt, D.A.; Vieira, R.; Norat, T. Dietary fiber and breast cancer risk: A systematic review and meta-analysis of prospective studies. Ann. Oncol. 2012, 23, 1394-1402. [CrossRef] 
39. Smith-Warner, S.A.; Spiegelman, D.; Yaun, S.S.; Adami, H.O.; Beeson, W.L.; van den Brandt, P.A.; Folsom, A.R.; Fraser, G.E.; Freudenheim, J.L.; Goldbohm, R.A.; et al. Intake of fruits and vegetables and risk of breast cancer a pooled analysis of cohort studies. J. Am. Med. Assoc. 2001, 285, 769-776. [CrossRef]

40. Song, J.K.; Bae, J.M. Citrus fruit intake and breast cancer risk: A quantitative systematic review. J. Breast Cancer 2013, 16, 72-76. [CrossRef]

41. Li, J.; Zou, L.; Chen, W.; Zhu, B.; Shen, N.; Ke, J.; Lou, J.; Song, R.; Zhong, R.; Miao, X. Dietary mushroom intake may reduce the risk of breast cancer: Evidence from a meta-analysis of observational studies. PLoS ONE 2014, 9, e93437. [CrossRef]

42. Seely, D.; Mills, E.J.; Wu, P.; Verma, S.; Guyatt, G.H. The effects of green tea consumption on incidence of breast cancer and recurrence of breast cancer: A systematic review and meta-analysis. Integr. Cancer Ther. 2005, 4, 144-155. [CrossRef] [PubMed]

43. Alexander, D.D.; Morimoto, L.M.; Mink, P.J.; Lowe, K.A. Summary and meta-analysis of prospective studies of animal fat intake and breast cancer. Nutr. Res. Rev. 2010, 23, 169-179. [CrossRef] [PubMed]

44. Boyd, N.F.; Stone, J.; Vogt, K.N.; Connelly, B.S.; Martin, L.J.; Minkin, S. Dietary fat and breast cancer risk revisited: A meta-analysis of the published literature. Br. J. Cancer 2003, 89, 1672-1685. [CrossRef] [PubMed]

45. Turner, L.B. A meta-analysis of fat intake, reproduction, and breast cancer risk: An evolutionary perspective. Am. J. Hum. Biol. 2011, 23, 601-608. [CrossRef] [PubMed]

46. Smith-Warner, S.A.; Spiegelman, D.; Adami, H.O.; Beeson, W.L.; van den Brandt, P.A.; Folsom, A.R.; Fraser, G.E.; Freudenheim, J.L.; Goldbohm, R.A.; Graham, S.; et al. Types of dietary fat and breast cancer: A pooled analysis of cohort studies. Int. J. Cancer 2001, 92, 767-774. [CrossRef]

47. Mulholland, H.G.; Murray, L.J.; Cardwell, C.R.; Cantwell, M.M. Dietary glycaemic index, glycaemic load and breast cancer risk: A systematic review and meta-analysis. Br. J. Cancer 2008, 99, 1170-1175. [CrossRef] [PubMed]

48. Mullie, P.; Koechlin, A.; Boniol, M.; Autier, P.; Boyle, P. Critical reviews in food science and nutrition relation between breast cancer and high glycemic index or glycemic load: A meta-analysis of prospective cohort studies relation between breast cancer and high glycemic index or glycemic load. Crit. Rev. Food Sci. Nutr. 2016, 56, 152-159. [CrossRef] [PubMed]

49. Yang, B.; Ren, X.-L.; Fu, Y.-Q.; Gao, J.-L.; Li, D. Ratio of n-3/n-6 PUFAs and risk of breast cancer: A meta-analysis of 274135 adult females from 11 independent prospective studies. BMC Cancer 2014, 14, 105. [CrossRef]

50. Zheng, J.-S.; Hu, X.-J.; Zhao, Y.-M.; Yang, J.; Li, D. Intake of fish and marine n-3 polyunsaturated fatty acids and risk of breast cancer: Meta-analysis of data from 21 independent prospective cohort studies. BMJ 2013, 346, F3706. [CrossRef]

51. Zhou, Y.; Wang, T.; Zhai, S.; Li, W.; Meng, Q. Linoleic acid and breast cancer risk: A meta-analysis. Public Health Nutr. 2015, 19, 1457-1463. [CrossRef]

52. Chen, P.; Hu, P.; Xie, D.; Qin, Y.; Wang, F.; Wang, H. Meta-analysis of vitamin D, calcium and the prevention of breast cancer. Breast Cancer Res. Treat. 2010, 121, 469-477. [CrossRef] [PubMed]

53. Gissel, T.; Rejnmark, L.; Mosekilde, L.; Vestergaard, P. Intake of vitamin D and risk of breast cancer-A. meta-analysis. J. Steroid Biochem. Mol. Biol. 2008, 111, 195-199. [CrossRef] [PubMed]

54. Cui, Y.; Rohan, T.E. Vitamin D, calcium, and breast cancer risk: A review. Cancer Epidemiol. Biomark. Prev. 2006, 15, 1427-1437. [CrossRef]

55. Chen, P.; Li, C.; Li, X.; Li, J.; Chu, R.; Wang, H. Higher dietary folate intake reduces the breast cancer risk: A systematic review and meta-analysis. Br. J. Cancer 2014, 110, 2327-2338. [CrossRef]

56. Larsson, S.C.; Giovannucci, E.; Wolk, A. Folate and risk of breast cancer: A. meta-analysis. J. Natl. Cancer Inst. 2007, 99, 64-76. [CrossRef]

57. Eichholzer, M.; Lüthy, J.; Moser, U.; Fowler, B. Folate and the risk of colorectal, breast and cervix cancer: The epidemiological evidence. Swiss Med. Wkly. 2001, 131, 539-549. [PubMed]

58. Hui, C.; Qi, X.; Qianyong, Z.; Xiaoli, P.; Jundong, Z.; Mantian, M. Flavonoids, flavonoid subclasses and breast cancer risk: A meta-analysis of epidemiologic studies. PLoS ONE 2013, 8, e54318. [CrossRef]

59. Hu, F.; Wang Yi, B.; Zhang, W.; Liang, J.; Lin, C.; Li, D.; Wang, F.; Pang, D.; Zhao, Y. Carotenoids and breast cancer risk: A meta-analysis and meta-regression. Breast Cancer Res. Treat. 2012, 131, 239-253. [CrossRef]

60. Rossi, R.E.; Pericleous, M.; Mandair, D.; Whyand, T.; Caplin, M.E. The role of dietary factors in prevention and progression of breast cancer. Anticancer Res. 2014, 34, 6861-6875. 
61. van den Brandt, P.A.; Schulpen, M. Mediterranean diet adherence and risk of postmenopausal breast cancer: Results of a cohort study and meta-analysis. Int. J. Cancer 2017, 140, 2220-2231. [CrossRef]

62. Campbell, K.L.; Foster-Schubert, K.E.; Alfano, C.M.; Wang, C.C.; Wang, C.Y.; Duggan, C.R.; Mason, C.; Imayama, I.; Kong, A.; Xiao, L. Reduced-calorie dietary weight loss, exercise, and sex hormones in postmenopausal women: Randomized controlled trial. J. Clin. Oncol. 2012, 30, 2314-2326. [CrossRef] [PubMed]

63. Pasanisi, P.; Berrino, F.; de Petris, M.; Venturelli, E.; Mastroianni, A.; Panico, S. Metabolic syndrome as a prognostic factor for breast cancer recurrences. Int. J. Cancer 2006, 119, 236-238. [CrossRef] [PubMed]

64. Frank, L.L.; Sorensen, B.E.; Yasui, Y.; Tworoger, S.S.; Schwartz, R.S.; Ulrich, C.M.; Irwin, M.L.; Rudolph, R.E.; Rajan, K.B.; Stanczyk, F.; et al. Effects of exercise on metabolic risk variables in overweight postmenopausal women: A randomized clinical trial. Obes. Res. 2005, 13, 615-625. [CrossRef] [PubMed]

65. McTiernan, A.; Tworoger, S.S.; Ulrich, C.M.; Yasui, Y.; Irwin, M.L.; Rajan, K.B.; Sorensen, B.; Rudolph, R.E.; Bowen, D.; Stanczyk, F.Z. Effect of exercise on serum estrogens in postmenopausal women: A 12-month randomized clinical trial. Cancer Res. 2004, 64, 2923-2928. [CrossRef]

66. Trichopoulou, C.; Bamia, P.L.; Trichopoulos, D. Conformity to traditional Mediterranean diet and breast cancer risk in the Greek EPIC (European Prospective Investigation into Cancer and Nutrition) cohort. Am. J. Clin. Nutr. 2010, 92, 620-625. [CrossRef]

67. Sieri, S.; Krogh, V.; Pala, V.; Muti, P.; Micheli, A.; Evangelista, A.; Tagliabue, G.; Berrino, F. Dietary patterns and risk of breast cancer in the ORDET cohort. Cancer Epidemiol. Biomark. Prev. 2004, 13, 567-572.

68. Murtaugh, M.A.; Sweeney, C.; Giuliano, A.R.; Herrick, J.S.; Hines, L.; Byers, T.; Baumgartner, K.B.; Slattery, M.L. Diet patterns and breast cancer risk in Hispanic and non-Hispanic white women: The Four-Corners Breast Cancer Study. Am. J. Clin. Nutr. 2008, 87, 978-984. [CrossRef]

69. Wu, H.; Yu, M.C.; Tseng, C.-C.; Stanczyk, F.Z.; Pike, M.C. Dietary patterns and breast cancer risk in Asian American women. Am. J. Clin. Nutr. 2009, 89, 1145-1154. [CrossRef]

70. Rose, D.P.; Goldman, M.; Connolly, J.M.; Strong, L.E. High-fiber diet reduces serum estrogen concentrations in premenopausal women. Am. J. Clin. Nutr. 1991, 54, 520-525. [CrossRef]

71. Surh, Y.-J. Cancer chemoprevention with dietary phytochemicals. Nat. Rev. Cancer 2003, 3, 768-780. [CrossRef]

72. Berrino, F. Il Cibo dell'Uomo. La Via della Salute tra Conoscenza Scientifica e Antiche Saggezze; Franco Angeli: Milan, Italy, 2015.

73. Masala, G.; Assedi, M.; Bendinelli, B.; Ermini, I.; Sieri, S.; Grioni, S.; Sacerdote, C.; Ricceri, F.; Panico, S.; Mattiello, A.; et al. Fruit and vegetables consumption and breast cancer risk: The EPIC Italy study. Breast Cancer Res. Treat. 2012, 132, 1127-1136. [CrossRef]

74. Ribaya-Mercado, J.D.; Blumberg, J.B. Lutein and zeaxanthin and their potential roles in disease prevention. J. Am. Coll. Nutr. 2004, 23, 567S-587S. [CrossRef] [PubMed]

75. Allegro, G.; Villarini, A. Prevenire i Tumori Mangiando con Gusto; Sperling \& Kupfer: Milan, Italy, 2013.

76. Wasser, S. Medicinal mushrooms as a source of antitumor and immunomodulating polysaccharides. Appl. Microbiol. Biotechnol. 2003, 60, 258-274.

77. Rohrmann, S.; Overvad, K.; Bueno-de-Mesquita, H.B.; Jakobsen, M.U.; Egeberg, R.; Tjønneland, A.; Nailler, L.; Boutron-Ruault, M.C.; Clavel-Chapelon, F.; Krogh, V.; et al. Meat consumption and mortality-Results from the European Prospective Investigation into Cancer and Nutrition. BMC Med. 2013, 11, 63. [CrossRef]

78. Ferretti, G.; Bacchetti, T.; Nègre-Salvayre, A.; Salvayre, R.; Dousset, N.; Curatola, G. Structural modifications of HDL and functional consequences. Atherosclerosis 2006, 184, 1-7. [CrossRef]

79. Aune, D.; De Stefani, E.; Ronco, A.L.; Boffetta, P.; Deneo-Pellegrini, H.; Acosta, G.; Mendilaharsu, M. Egg consumption and the risk of cancer: A multisite case-control study in Uruguay. Asian Pacific J. Cancer Prev. 2009, 10, 869-876.

80. Li, F.; Zhou, Y.; Hu, R.T.; Hou, L.N.; Du, Y.J.; Zhang, X.J.; Olkkonen, V.M.; Tan, W.L. Egg consumption and risk of bladder cancer: A meta-analysis. Nutr. Cancer 2013, 65, 538-546. [CrossRef]

81. Lu, L.J.; Anderson, K.E.; Grady, J.J.; Nagamani, M. Effects of soya consumption for one month on steroid hormones in premenopausal women: Implications for breast cancer risk reduction. Cancer Epidemiol. Prev. Biomark. 1996, 5, 63-70.

82. Lu, L.-J.W.; Anderson, K.E.; Grady, J.J.; Kohen, F.; Nagamani, M. Decreased ovarian hormones during a soya diet: Implications for breast cancer prevention. Cancer Res. 2000, 60, 4112-4121. 
83. Bartolozzi, G. Fisiopatologia della vitamina D (seconda parte). Medico. E. Bambino. Pagine. Elettron. 2007, 10, $1-3$.

84. Pasanisi, P.; Bruno, E.; Venturelli, E.; Manoukian, S.; Barile, M.; Peissel, B.; De Giacomi, C.; Bonanni, B.; Berrino, J.; Berrino, F. Serum levels of IGF-I and BRCA penetrance: A case control study in breast cancer families. Fam. Cancer 2011, 10, 521-528. [CrossRef] [PubMed]

85. Norat, T.; Dossus, L.; Rinaldi, S.; Overvad, K.; Grønbaek, H.; Tjønneland, A.; Olsen, A.; Clavel-Chapelon, F.; Boutron-Ruault, M.C.; Boeing, H. Diet, serum insulin-like growth factor-I and IGF-binding protein-3 in European women. Eur. J. Clin. Nutr. 2007, 61, 91-98. [CrossRef] [PubMed]

86. Qin, L.-Q.; He, K.; Xu, J.-Y. Milk consumption and circulating insulin-like growth factor-I level: A systematic literature review. Int. J. Food Sci. Nutr. 2009, 60, 330-340. [CrossRef] [PubMed]

87. Silalahi, J. Anticancer and health protective properties of citrus fruit components. Asia Pac. J. Clin. Nutr. 2007, 11, 79-84. [CrossRef]

88. Boeke, C.E.; Tamimi, R.M.; Berkey, C.S.; Colditz, G.A.; Eliassen, A.H.; Malspeis, S.; Willett, W.C.; Frazier, A.L. Adolescent carotenoid intake and benign breast disease. Pediatrics 2014, 133, 1-7. [CrossRef]

89. Cui, Y.; Lu, Z.; Bai, L.; Shi, Z.; Zhao, W.e.; Zhao, B. $\beta$-Carotene induces apoptosis and up-regulates peroxisome proliferator-activated receptor $\gamma$ expression and reactive oxygen species production in MCF-7 cancer cells. Eur. J. Cancer 2007, 43, 2590-2601. [CrossRef]

90. Hui, C.; Yujie, F.; Lijia, Y.; Long, Y.; Hongxia, X.; Yong, Z.; Jundong, Z.; Qianyong, Z.; Mantian, M. MicroRNA-34a and microRNA-21 play roles in the chemopreventive effects of 3,6-dihydroxyflavone on 1-methyl-1-nitrosourea-induced breast carcinogenesis. Breast Cancer Res. 2012, 14, R80. [CrossRef]

91. Zhang, S.; Yang, X.; Coburn, R.A.; Morris, M.E. Structure activity relationships and quantitative for the flavonoid-mediated inhibition of breast cancer resistance protein. Biochem. Pharmacol. 2005, 70, 627-639. [CrossRef]

92. Neuhouser, M.L. Review. Dietary flavonoids and cancer risk: Evidence from human population studies. Nutr. Cancer 2004, 50, 16-22. [CrossRef]

93. Ross, J.A.; Kasum, C.M. Dietary flavonoids: Bioavailability, metabolic effects, and safety. Annu. Rev. Nutr. 2002, 22, 19-34. [CrossRef]

94. Papa, V.; Belfiore, A. Insulin receptors in breast cancer: Biological and clinical role. J. Endocrinol. Investig. 1994, 19, 324-333. [CrossRef] [PubMed]

95. Laamiri, F.Z.; Otmani, A.; Ahid, S.; Barkat, A. Lipid profile among Moroccan overweight women and breast cancer: A case-control study. Int. J. Gen. Med. 2013, 6, 439-445.

96. Sieri, S.; Krogh, V.; Ferrari, P.; Berrino, F.; Pala, V.; Thiébaut, A.C.; Tjønneland, A.; Olsen, A.; Overvad, K.; Jakobsen, M.U.; et al. Dietary fat and breast cancer risk in the European Prospective Investigation into Cancer and Nutrition. Am. J. Clin. Nutr. 2008, 88, 1304-1312. [PubMed]

97. Armstrong, B.; Doll, R. Environmental factors and cancer incidence and mortality in different countries, with special reference to dietary practices. Int. J. Cancer 1975, 15, 617-631. [CrossRef] [PubMed]

98. World Health Organization. Obesity and Overweight. Global Strategy on Diet, Physical Activity and Health. Available online: https://www.who.int/dietphysicalactivity/publications/obesity/en/ (accessed on 29 June 2020).

99. Golay, A.; Bobbioni, E. The role of dietary fat in obesity. Int. J. Obes. Relat. Metab. Disord. 1997, 21, S2-S11. [PubMed]

100. Astrup, A.; Buemann, B.; Western, P.; Toubro, S.; Raben, A.; Christensen, N.J. Obesity as an adaptation to a high-fat diet: Evidence from a cross-sectional study. Am. J. Clin. Nutr. 1994, 59, 350-355. [CrossRef]

101. Jequier, E. Pathways to obesity. Int. J. Obes. 2002, 26, S18-S27. [CrossRef] [PubMed]

102. Hill, J.O.; Melanson, E.L.; Wyatt, H.T. Dietary fat intake and regulation of energy balance: Implications for obesity. J. Nutr. 2000, 130, 284S-288S. [CrossRef]

103. Bray, G.A.; Popkin, B.M. Dietary fat intake does affect obesity! Am. J. Clin. Nutr. 1998, 68, 1157-1173. [CrossRef] [PubMed]

104. Schrauwen, P.; Westerterp, K.R. The role of high-fat diets and physical activity in the regulation of body weight. Br. J. Nutr. 2000, 84, 417-427. [CrossRef] [PubMed]

105. Larsson, S.C.; Wolk, A. Excess body fatness: An important cause of most cancers. Lancet 2008, 371, 536-537. [CrossRef] 
106. Dossus, L.; Kaaks, R. Nutrition, metabolic factors and cancer risk. Best Pract. Res. Clin. Endocrinol. Metab. 2008, 22, 551-571. [CrossRef]

107. Key, T.J.; Allen, N.E.; Spencer, E.A.; Travis, R.C. Nutrition and breast cancer. The Breast 2003, 12, $412-416$. [CrossRef]

108. Lahmann, P.H.; Lissner, L.; Berglund, G. Breast cancer risk in overweight postmenopausal women. Cancer Epidemiol. Prev. Biomark. 2004, 13, 8.

109. Murff, H.J.; Shu, X.O.; Li, H.; Yang, G.; Wu, X.; Cai, H.; Wen, W.; Gao, Y.T.; Zheng, W. Dietary polyunsaturated fatty acids and breast cancer risk in Chinese women: A prospective cohort study. Int. J. Cancer 2011, 128, 1434-1441. [CrossRef] [PubMed]

110. Liu, J.; Ma, D.W.L. The role of n-3 polyunsaturated fatty acids in the prevention and treatment of breast cancer. Nutrients 2014, 6, 5184-5223. [CrossRef]

111. Corso, G.; Maisonneuve, P.; Massari, G.; Invento, A.; Pravettoni, G.; De Scalzi, A.; Intra, M.; Galimberti, V.; Morigi, C.; Lauretta, M.; et al. Validation of a Novel Nomogram for Prediction of Local Relapse after Surgery for Invasive Breast Carcinoma. Ann. Surg. Oncol. 2020, 27, 1864-1874. [CrossRef] [PubMed]

112. World Cancer Research Fund/American Institute for Cancer Research. Diet, Nutrition, Physical Activity and Breast Cancer. Continuous Update Project Expert Report. 2018. Available online: https:/www.wcrf.org/ sites/default/files/Summary-of-Third-Expert-Report-2018.pdf (accessed on 29 June 2020).

(C) 2020 by the authors. Licensee MDPI, Basel, Switzerland. This article is an open access article distributed under the terms and conditions of the Creative Commons Attribution (CC BY) license (http://creativecommons.org/licenses/by/4.0/). 